\title{
Critical dynamics of non-conserved strongly anisotropic permutation symmetric three-vector model
}

\author{
Rajiv G. Pereira \\ School of Physics, IISER Thiruvananthapuram, Vithura, Kerala 695551, India
}

\begin{abstract}
We explore, employing the renormalization-group theory, the critical scaling behavior of the permutation symmetric three-vector model that obeys non-conserving dynamics and has a relevant anisotropic perturbation which drives the system into a non-equilibrium steady state. We explicitly find the independent critical exponents with corrections up to two loops. They include the static exponents $\nu$ and $\eta$, the off equilibrium exponent $\widetilde{\eta}$, the dynamic exponent $z$ and the strong anisotropy exponent $\Delta$. We also express the other anisotropy exponents in terms of these.
\end{abstract}

\section{INTRODUCTION}

Universality exhibited by systems out of equilibrium has been a prominent object of study in statistical physics, especially since the formulation of the renormalization-group $(R G)$ theory [1 4]. A variety of genuine non-equilibrium $(N E)$ universality classes have been identified and well studied in the past few decades. The driven diffusive systems [2, 5, 6] and the percolation models [7, 8] are a few examples. However, in comparison to equilibrium and near-equilibrium classes [9, 10, genuine non-equilibrium ones remain far less explored.

Driven-diffusive models constitute an important category that violates the detailed-balance condition. They have been widely used to describe physical systems, such as fast ionic conductors 11, 12 and traffic jams 13, 14, in order to investigate physics far from equilibrium. A variety of such models have been explored in the past [5], and they continue to appear in recent studies, for instance, Bose condensation transition [15, 16 and systems coupled to mutually interacting Langmuir kinetics [17]. These are essentially Ising-like models with anisotropic forces, and many of them exhibit universality distinct from that of any equilibrium class [18 20]. However, those models with spatially biased forces that violate detailed-balance even at the long-distance and large-time limit are mostly the ones that follow conserving dynamics.

Non-conserved Ising-like systems with relevant anisotropic perturbations are rare, and their critical properties are far less explored. One such exception can be found in Ref. [21, wherein a cyclic permutation symmetric three-vector model with non-conserving dynamics and anisotropic perturbations was introduced. It was shown that for this model, below the critical dimension $d_{c}=4$, there exists an infrared stable fixed point at which one of the anisotropic perturbations is relevant, thus identifying a new genuine non-equilibrium universality class.

Though the anisotropic $N E$ fixed point was identified, the critical behavior of this class has not been investigated. Secondly, the relevance of the anisotropic term should reflect as difference in the longitudinal and the transverse power-law behavior of the correlation functions. Further, unlike other commonly found Ising-like systems with relevant spatial bias [18, 20, 22, 23], this model follows non-conserving dynamics. These factors raise several interesting questions. What are the similarities and the differences in the critical behavior of the model from that of the conserved ones? Does the model exhibit common critical features such as faster decay of longitudinal fluctuations [5]? Is the critical power-law decay of the response and the correlation functions spatially biased? Are the $\eta$-like exponents in the real space different from those in the momentum space?

Motivated by these questions, we explore the critical scaling behavior of this class. For this, we look at a simpler model obtained by replacing the cyclic permutation symmetry in the model introduced in Ref. 21] by permutation symmetry. In other words, we consider the non-conserved strongly anisotropic permutation symmetric $(N S A P S)$ three-vector model. It is sufficient to study this model and determine the critical exponents as it has the same $N E$ fixed point. A nontrivial correction to two of the independent exponents, the strong anisotropy exponent $\Delta$ and the correlation length exponent $\nu$, can be obtained at the one-loop order. However, there are other exponents, where a non-trivial correction appears only at the two-loop order. Hence we renormalize the theory to this order.

We organize this paper as follows. In Sec. II] we introduce the NSAPS three-vector model. In Sec. III, we first discuss the renormalization of the theory and then briefly describe the computational methods employed in the two-loop calculation. In Sec. IV] we obtain the critical exponents to two-loop order in an expansion around the upper critical dimension $d_{c}=4$ and then discuss the various critical features of the model. In Appendix A, the computational methods used in obtaining and evaluating the Feynman diagrams are detailed, and in Appendices B to I] the relevant $1 \mathrm{PI}$ diagrams and their divergences are listed.

\section{THE MODEL}

The most general field theory for non-conserved $N$ vector models subject to anisotropic forces with all the marginal perturbations in $4+1$ dimensions, was constructed in Ref [21. The theory is written in Martin- 
Siggia-Rose (MSR) formalism [24] as

$$
\begin{array}{r}
\mathcal{S}(\phi, \tilde{\phi})=\int_{x}\left[\widetilde{\phi}_{a}\left(\partial_{t}-\nabla^{2}+r\right) \phi_{a}-\frac{1}{2} \mathcal{E}_{a b c} \widetilde{\phi}_{a} \phi_{b} \partial_{\|} \phi_{c}\right. \\
\left.+\frac{1}{3 !} G_{a b c d} \widetilde{\phi}_{a} \phi_{b} \phi_{c} \phi_{d}-T \widetilde{\phi}_{a} \widetilde{\phi}_{a}\right],
\end{array}
$$

where $x$ denotes the time and the space coordinates $\{t, \boldsymbol{x}\}, \int_{x} \equiv \int d t d^{d} \boldsymbol{x}, \tilde{\phi}$ is the auxillary field and $T$ is the noise strength. The fields $\phi$ and $\tilde{\phi}$ are functions of $x$ and the repeated indices are summed over.

It was shown in Ref. 21] that only when the number of components $N=3$, there can be anisotropic perturbations consistent with a single length scale. In the case of cyclic permutation symmetry, there are five allowed independent couplings namely, $G_{1111}, G_{1122}, G_{1133}, \mathcal{E}_{123}$ and $\mathcal{E}_{132}$. Below the upper critical dimension $d_{c}=4$ this model has an infrared stable $N E$ fixed point at which the anisotropic coupling $\mathcal{E}_{123}$ and the couplings $G_{1111}$ and $G_{1122}$ are relevant, while the couplings $G_{1133}$ and $\mathcal{E}_{132}$ are irrelevant 21. Thus, a non-conserved Isinglike model with a relevant anisotropic perturbation was constructed, identifying a new genuine non-equilibrium universality class.

If we now restrict to full permutation symmetry, the NSAPS three-vector model is obtained, where the number of allowed independent couplings reduce to three namely, $G_{1111}, G_{1122}$ and $\mathcal{E}_{123}$. This model has the same infrared stable fixed point as the cyclic permutation symmetric one [21. Therefore, it is sufficient to study the critical scaling behavior of this model. The MSR action for this simpler case can be written as

$$
\begin{array}{r}
S=\sum_{a=1}^{3} \int_{x}\left[\widetilde{\phi}_{a}\left(\partial_{t}-D\left(\nabla_{\perp}^{2}+\rho \partial_{\|}^{2}-r\right)\right) \phi_{a}-T \widetilde{\phi}_{a}^{2}\right. \\
\left.+\frac{u_{0}}{3 !} \widetilde{\phi}_{a} \phi_{a}^{3}+\frac{u_{1}}{2 !} \widetilde{\phi}_{a} \phi_{a}\left(\phi_{a+1}^{2}+\phi_{a+2}^{2}\right)+e_{p} \phi_{a+1} \phi_{a+2} \partial_{\|} \widetilde{\phi}_{a}\right],
\end{array}
$$

where $u_{0} \equiv G_{1111}, u_{1} \equiv G_{1122}, e_{p} \equiv \mathcal{E}_{123}, \phi_{i+3} \equiv \phi_{i}$ and $\widetilde{\phi}_{i+3} \equiv \widetilde{\phi}_{i}$. We split the $\nabla^{2}$ term into the longitudinal and the transverse components by introducing the coefficient $\rho$ as the theory is spatially anisotropic.

We proceed to perform a two-loop $R G$ analysis on the NSAPS three-vector model and extract the critical exponents associated to the response and the correlation functions.

\section{RG ANALYSIS}

In this section, we first discuss the standard renormalization procedure (see, for example, the excellent text book by Tauber [2]), and apply it to the NSAPS threevector model, where we define the renormalization constants and state the renormalization conditions. Then we briefly describe the computational techniques employed in the calculation, which are suitable when the diagrams are numerous. The computational packages FeynArts 25] and FeynCalc [26, 27] are used with Mathematica 28 to obtain the Feynman diagrams and the package SecDec [29] is used for numerical dimensional regularization.

\section{Definitions and notations}

The effective action is written as

$\Gamma[\psi, \widetilde{\psi}]=-\ln \mathcal{Z}[J, \widetilde{J}]+\sum_{a} \int_{x} J_{a}(x) \psi_{a}(x)+\widetilde{J}_{a}(x) \widetilde{\psi}_{a}(x)$,

where $\psi(x)=\frac{\delta \ln \mathcal{Z}}{\delta J(x)}, \quad \widetilde{\psi}(x)=\frac{\delta \ln \mathcal{Z}}{\delta \widetilde{J}(x)}$, and the generating functional for correlation functions $\mathcal{Z}[J, \widetilde{J}]=$ $\left\langle\exp \sum_{a} \int_{x} \phi_{a}(x) J_{a}(x)+\widetilde{\phi}_{a}(x) \widetilde{J}_{a}(x)\right\rangle$. The 1PI diagrams are obtained by taking the functional derivaties of $\Gamma$,

$$
\begin{aligned}
\Gamma_{\widetilde{n}, n}^{\widetilde{a}_{1} \ldots \widetilde{a}_{\tilde{n}} a_{1} \ldots a_{n}}\left(\widetilde{x}_{1}, . . \widetilde{x}_{\widetilde{n}} ; x_{1}, \ldots x_{n}\right) & = \\
& \left.\prod_{i=1}^{\tilde{n}} \frac{\delta}{\delta \widetilde{\psi}_{\widetilde{a}_{i}}\left(\widetilde{x}_{i}\right)} \prod_{j=1}^{n} \frac{\delta}{\delta \psi_{a_{j}}\left(x_{j}\right)} \Gamma[\widetilde{\psi}, \psi]\right|_{\tilde{\psi}=\psi=0}
\end{aligned}
$$

The ultraviolet divergences are absorbed into the renormalization constants $Z_{\phi}, Z_{\widetilde{\phi}}, Z_{D}, Z_{\rho}, Z_{T}, Z_{0}, Z_{1}$ and $Z_{p}$, and the bare fields and the bare parameters are written in terms of their renormalized counterparts as

$$
\begin{array}{r}
\phi_{a}=Z_{\phi}^{1 / 2} \phi_{a R}, \widetilde{\phi}_{a}=Z_{\widetilde{\phi}}^{1 / 2} \widetilde{\phi}_{a R}, D=\frac{Z_{D}}{Z} D_{R}, \\
\rho=\frac{Z_{\rho}}{Z_{D}} \rho_{R}, T=\frac{Z_{T}}{Z_{\widetilde{\phi}}} T_{R}, r=\frac{Z_{r}}{Z_{D}} \mu^{2} r_{R}, \\
u_{0}=\frac{Z_{0}}{Z Z_{\phi}} u_{0 R}, u_{1}=\frac{Z_{1}}{Z Z_{\phi}} u_{1 R}, \quad e_{p}=\frac{Z_{p}}{Z Z_{\phi}{ }^{1 / 2}} e_{p R},
\end{array}
$$

where $Z=\sqrt{Z_{\phi} Z_{\widetilde{\phi}}}$, the subscript $R$ denontes the renormalized quantities and the factor $\mu$ is introduced to make $r_{R}$ dimensionless. The renormalization constants are fixed by the following renormalization condtions with the 
minimal substraction scheme.

$$
\begin{aligned}
& \Gamma_{R 1,1}^{11}\left(q_{i}=0\right)=D_{R} r_{R} \mu^{2}, \\
& \left.\frac{\partial}{\partial q_{\|}^{2}} \Gamma_{R 1,1}^{11}(q ; q)\right|_{q=0}=D_{R} \rho_{R}, \\
& \left.\frac{\partial}{\partial i q_{0}} \Gamma_{R 1,1}^{11}(q ; q)\right|_{q=0}=1, \\
& \left.\frac{\partial}{\partial q_{\perp}^{2}} \Gamma_{R 1,1}^{11}(q ; q)\right|_{q=0}=D_{R}, \\
& \Gamma_{R 2,0}^{11}\left(q_{i}=0\right)=-2 T_{R}, \quad \\
& \left.\frac{\partial}{\partial i q_{\|}} \Gamma_{R}^{123}{ }_{1,2}^{11}\left(-q, \frac{q}{2}, \frac{q}{2}\right)\right|_{q=0}=e_{p R}, \\
& \Gamma_{R}^{1111}{ }_{1,3}\left(q_{i}=0\right)=u_{0 R}, \\
& \Gamma_{R}^{1122}{ }_{1,3}\left(q_{i}=0\right)=u_{1 R} .
\end{aligned}
$$

\section{Diagramatics and perturbative computation}

The unperturbed action in Fourier space is

$$
S=\sum_{a} \int_{q} \widetilde{\phi}_{a}(-q)\left(-i q_{0}+M(\boldsymbol{q})\right) \phi_{a}(q),
$$

where $M(\boldsymbol{q})=D\left(\boldsymbol{q}_{\perp}{ }^{2}+\rho q_{\|}^{2}+r\right)$ and $\int_{q} \equiv \frac{1}{(2 \pi)^{d+1}} \int d q_{0} d \boldsymbol{q}$. The Fourier transform of a function $f(x)$ is defined by the relation $f(x)=\int_{q} f(q) e^{-i q \cdot x}$, where $q \cdot x=q_{0} x_{0}-\boldsymbol{q} \cdot \boldsymbol{x}$. The subscripts $\perp, \|$ and 0 denote the transverse, the longitudnal and the temporal directions, respectively.

The two non-vanishing unperturbed two-point correlations are

$$
\begin{gathered}
\left\langle\phi_{a}\left(q_{1}\right) \widetilde{\phi}_{b}\left(q_{2}\right)\right\rangle_{0}=\frac{\delta_{a b} \bar{\delta}\left(q_{1}+q_{2}\right)}{-i q_{0}+M(\boldsymbol{q})}=\delta_{a b} \bar{\delta}\left(q_{1}+q_{2}\right) G_{0}\left(q_{1}\right), \\
\left\langle\phi_{a}\left(q_{1}\right) \phi_{b}\left(q_{2}\right)\right\rangle_{0}=2 T \frac{\delta_{a b} \bar{\delta}\left(q_{1}+q_{2}\right)}{q_{0}^{2}+M(\boldsymbol{q})^{2}}=\delta_{a b} \bar{\delta}\left(q_{1}+q_{2}\right) C_{0}\left(q_{1}\right),
\end{gathered}
$$

where $\bar{\delta}(q) \equiv(2 \pi)^{d+1} \delta(q)$.

The diagrammatic representations of the two-point Gaussian correlation functions and the perturbations are illustrated in Fig. 1. With the help of these building blocks, we perform the perturbative expansions of the vertex functions to two loops and extract the divergences. This is implemented computationally in the following steps.

1. For a given vertex function, we obtain all the contributing Feynman diagrams and the corresponding expressions to two-loops using the packages FeynArts [25] and FeynCalc [26, 27. For this, we first draw all the topologically distinct irreducible diagrams (topologies) with $l$ external legs that can be constructed with three-point and four-point vertices to two loops, where $l$ is determined by the
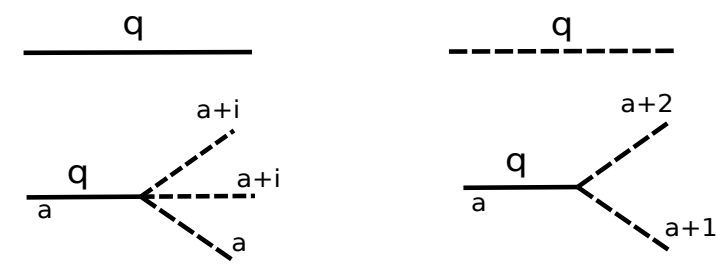

FIG. 1. The straight line represents $G_{0}(q)$ and the dotted line $C_{0}(q)$. The four-point vertex takes the value $u_{0} / 6$ if $i=0$ and $u_{1} / 2$ if $i \neq 0$. The three-point vertex takes the value $i q_{\|} e_{p}$. The dotted branch becomes a straight line when it is hit with an auxiliary field and remains dotted otherwise. We choose the convention that the external $\phi$ fields hit from the left and the external $\widetilde{\phi}$ fields hit from the right. This makes the arrow which is usually attached to the propagator redundant and is hence not explicitly shown.

vertex function we evaluate. Then, we draw all possible realizations of the topologies that can be obtained using the building blocks shown in Fig. 1. Each such realization (Feynman diagram) correspond to an expression of the form

$$
A_{f} \int_{\left\{q_{i}\right\}} p_{1_{\|}} p_{2_{\|}} \ldots G_{0}\left(k_{1}\right) G_{0}\left(k_{2}\right) \ldots C_{0}\left(k_{m}\right) C_{0}\left(k_{m+1}\right) \ldots
$$

where $\left\{q_{i}\right\}$ is the set of internal momenta, $p_{i}$ and $k_{i}$ are in general linear combinations of the internal and the external momenta and $A_{f}$ is the overall factor associated with each diagram.

2. Derivatives and limits are now applied to isolate the divergences in the expressions obtained in step 1 . Integrating out $q_{i 0}$ s subsequently leads to an expression of the form

$$
B_{f} \int_{\left\{\boldsymbol{q}_{i}\right\}} \frac{p_{1 \|}^{m_{1}}\left|\boldsymbol{p}_{2 \perp}\right|^{m_{2}}}{\mathcal{M}_{1}^{n_{1}} \mathcal{M}_{2}^{n_{2}} \cdots}+\text { similar terms }
$$

where $\mathcal{M}_{i}$ is either $M\left(\boldsymbol{k}_{i}\right)$ or sum of $M\left(\boldsymbol{k}_{i}\right)$ s. The variables $\boldsymbol{p}_{i}$ and $\boldsymbol{k}_{i}$ now contain only the internal momenta. Once the parallel components of $q_{i}$ s are scaled approprietly, $M\left(k_{i}\right)$ takes the form of the scalar propagator with a factor $D$.

3 . The integrals obtained by the above procedure are regularized by the method of dimensional regularization and the UV-divergent parts are expanded as

$$
\hat{\mathcal{D}}\left(\int_{\left\{\boldsymbol{q}_{\boldsymbol{i}}\right\}} \frac{p_{1 \|}^{m_{1}}\left|\boldsymbol{p}_{\boldsymbol{2}_{\perp}}\right|^{m_{2}}}{\mathcal{M}_{1}^{n_{1}} \mathcal{M}_{2}^{n_{2}} \ldots}\right)=\frac{\omega_{1}}{\epsilon^{2}}+\frac{\omega_{2}}{\epsilon},
$$

where the operator $\hat{D}$ is defined such that $\hat{D}$ acting on an intergral gives the poles of the corresponding dimensionally regularized integral, the parameter 
$\epsilon=4-d$, and $\omega_{1}$ and $\omega_{2}$ are real numbers. This step is implemented with the help of the package SecDec [29]31.

The above steps are elucidated with the help of an example in Appendix A.

4. Once all the diagrams contributing to a given vertex function are evaluated and the divergences are obtained in negative powers of $\epsilon$, we apply the renormalization conditions with the minimal subtraction scheme to obtain the renormalization constants.

The divergent 1PI Feynman diagrams contributing to the various relevant vertex functions, and the renormalization constants are given in Appendices B] to I

There are 11 distinct divergent Feynman diagrams contributing to the various relevant vertex functions at the one-loop order. The presence of both three-point and four-point vertices enhances the number of diagrams at the two-loop order enormously, where the number rises to 319 . In the absence of the four-point vertices, the total number of two-loop diagrams reduces to 27 , while in the absence of the three-point vertices, the total number of two-loop diagrams reduces to 19 . For instance, there are 25 two-loop diagrams contributing to $\Gamma_{1,1}^{11}(-q ; q)$, as shown in Fig. 3, of which only 6 diagrams (diagrams 10 to 15) are constructed with three-point vertices alone, while only two diagrams (diagrams 20 and 25) are constructed with four-point vertices alone. Similarily, of the 83 diagrams contributing to $\left.\frac{\partial}{\partial i q_{\|}} \Gamma_{1,2}^{123}\left(-q, \frac{q}{2}, \frac{q}{2}\right)\right|_{q=0}$, shown in Table IX, only 21 diagrams (diagrams 14 to 25 and 73 to 81 ) are constructed with three-point vertices alone, while none is constructed with four-point vertices alone. Of the 94 diagrams contributing to $\Gamma_{1,3}^{1111}\left(q_{i}=0\right)$, shown in Table XII none is constructed with three-point vertices alone, while only 8 diagrams (diagrams 47 to 53 and 94) are constructed with four-point vertices alone. Of the 116 diagrams contributing to $\Gamma_{1,3}^{1122}\left(q_{i}=0\right)$, shown in Table XV none is constructed with three-point vertices alone and only 8 diagrams (diagrams 67 to 73 and 116) are constructed with four-point vertices alone. The only one diagram contributing to $\Gamma_{2,0}^{11}(0)$, shown in Table VII. is constructed with four-point vertices alone.

\section{THE CRITICAL EXPONENTS OF THE NSAPS THREE-VECTOR MODEL}

We proceed to write down and solve the $R G$ equation to obtain the scaling form of the vertex functions at the $N E$ fixed point as the temperature approaches the critical value. In particular, we analyze the scaling behavior of the dynamic structure factor and the dynamic susceptibility and extract the exponents associated with them.

For notational simplicity, the subscript $R$ is suppressed in this section, and the following dimensionless couplings are employed

$$
\begin{aligned}
& \lambda_{0}=\frac{1}{8 \pi^{2}} \frac{T}{D^{2} \rho^{1 / 2}} u_{0} \mu^{-\epsilon}, \\
& \lambda_{1}=\frac{1}{8 \pi^{2}} \frac{T}{D^{2} \rho^{1 / 2}} u_{1} \mu^{-\epsilon}, \\
& \lambda_{2}=\frac{1}{8 \pi^{2}} \frac{T}{D^{3} \rho^{3 / 2}} e_{p}^{2} \mu^{-\epsilon} .
\end{aligned}
$$

The beta functions are

$$
\beta_{i}=\mu \frac{d \lambda_{i}}{d \mu}
$$

for $i=0,1$ and 2, and Wilson's flow functions are

$$
\begin{aligned}
& \gamma_{\phi}=-\mu \frac{\partial}{\partial \mu} \ln Z_{\phi}, \gamma_{\tilde{\phi}}=-\mu \frac{\partial}{\partial \mu} \ln Z_{\tilde{\phi}}, \gamma_{D}=\mu \frac{\partial}{\partial \mu} \ln D \\
& \gamma_{\rho}=\mu \frac{\partial}{\partial \mu} \ln \rho, \gamma_{T}=\mu \frac{\partial}{\partial \mu} \ln T, \gamma_{r}=\mu \frac{\partial}{\partial \mu} \ln r
\end{aligned}
$$

where the derivatives are to be taken keeping the bare parameters and couplings constant. Since all the UV divergences can be absorbed into the eight renormalization constants $Z_{\phi}, Z_{D}, Z_{\rho}, Z_{T}, Z_{r}, Z_{0}, Z_{1}$ and $Z_{p}$, the auxiliary field renormalization constant $Z_{\widetilde{\phi}}$ is set to unity which implies that $\gamma_{\widetilde{\phi}}=0$. of $\mu$,

We now write down the $R G$ equation, which follows from the fact that the bare vertex functions are independent

$$
\left[\mu \frac{\partial}{\partial \mu}+\gamma_{\phi} \frac{n}{2}+\sum_{i} \gamma_{s_{i}} s_{i} \frac{\partial}{\partial s_{i}}+\sum_{i} \beta_{i} \frac{\partial}{\partial \lambda_{i}}\right] \Gamma_{\widetilde{n}, n}\left(q_{i}, s_{i}, \lambda_{i}, \mu\right)=0
$$

where $q_{i}$ denotes the external momenta and $s_{i}$ denotes the elements of the set of parameters $\{D, \rho, T, r\}$.

The beta functions are obtained by using the renormalization constants given in Appendices B to in equations (B3), 
(C1), (D1), (E1), (F1), (G1), (H1) and (I1), together with Eq. 20), and are explicitly written as

$$
\begin{aligned}
\beta_{0}= & -\epsilon \lambda_{0}+1.5 \lambda_{0}^{2}+3 \lambda_{1}^{2}+0.375 \lambda_{0} \lambda_{2}-1.41667 \lambda_{0}^{3}-6 \lambda_{1}^{3}+0.336482 \lambda_{2} \lambda_{0}^{2}-2.5 \lambda_{1}^{2} \lambda_{0}+0.156108 \lambda_{2}^{2} \lambda_{0} \\
& -0.341841 \lambda_{0} \lambda_{1} \lambda_{2}-0.0163937 \lambda_{1} \lambda_{2}^{2}+0.991439 \lambda_{1}^{2} \lambda_{2}, \\
\beta_{1}= & -\epsilon \lambda_{1}+2.5 \lambda_{1}^{2}+\lambda_{0} \lambda_{1}+0.375 \lambda_{1} \lambda_{2}-4.5 \lambda_{1}^{3}-0.0359603 \lambda_{0}^{2} \lambda_{2}-3 \lambda_{0} \lambda_{1}^{2}+0.700121 \lambda_{2} \lambda_{1}^{2}-0.416667 \lambda_{0}^{2} \lambda_{1} \\
& +0.158841 \lambda_{2}^{2} \lambda_{1}+0.135281 \lambda_{0} \lambda_{2} \lambda_{1}-0.00149424 \lambda_{0} \lambda_{2}^{2}, \\
\beta_{2}= & -\epsilon \lambda_{2}+1.125 \lambda_{2}^{2}+3.5 \lambda_{1} \lambda_{2}+0.475028 \lambda_{2}^{3}-0.181794 \lambda_{0} \lambda_{2}^{2}+1.38638 \lambda_{1} \lambda_{2}^{2}+0.125 \lambda_{0}^{2} \lambda_{2}-4.05652 \lambda_{1}^{2} \lambda_{2} \\
& -2.90455 \lambda_{0} \lambda_{1} \lambda_{2} .
\end{aligned}
$$

Similarly, we obtain Wilson's flow functions by using the renormalization constants given in the Appendices B to 1 together with Eq. 21) and are explicitly written as

$$
\begin{aligned}
\gamma_{\phi} & =-0.143841 \lambda_{0}^{2}-0.863046 \lambda_{1}^{2} \\
\gamma_{D} & =-0.0416667 \lambda_{0}^{2}-0.25 \lambda_{1}^{2}-0.025463 \lambda_{1} \lambda_{2} \\
\gamma_{\rho} & =-0.75 \lambda_{2}-0.0416667 \lambda_{0}^{2}-0.25 \lambda_{1}^{2}-0.312217 \lambda_{2}^{2}-0.308408 \lambda_{1} \lambda_{2}, \\
\gamma_{T} & =-0.0719205 \lambda_{0}^{2}-0.431523 \lambda_{1}^{2} \\
\gamma_{r} & =-2+0.5 \lambda_{0}+\lambda_{1}-0.25 \lambda_{0}^{2}-1.5 \lambda_{1}^{2}+0.112161 \lambda_{2} \lambda_{0}+0.224321 \lambda_{1} \lambda_{2}
\end{aligned}
$$

The set of equations

$$
\beta_{i}=0
$$

leads to the critical points. For $\epsilon<0$, the equilibrium Gaussian fixed point is stable. For $\epsilon>0$, the following $N E$ fixed point is stable,

$$
\begin{aligned}
& \lambda_{0}{ }^{*}=0.461538 \epsilon+0.173639 \epsilon^{2}, \\
& \lambda_{1}{ }^{*}=0.153847 \epsilon+0.0837608 \epsilon^{2}, \\
& \lambda_{2}{ }^{*}=0.410255 \epsilon-0.133947 \epsilon^{2},
\end{aligned}
$$

where the superscript ${ }^{*}$ denotes the fixed point values of the couplings $\lambda_{i}$. The above result agrees with the one-loop calculations in Ref. 21] to that order. By substituting Eq. 26) in Eq. (24), we further obtain Wilson's flow functions at this fixed point as

$$
\begin{aligned}
& \gamma_{D}{ }^{*}=-0.0164001 \epsilon^{2}, \\
& \gamma_{\rho}^{*}=-0.307691 \epsilon+0.0136525 \epsilon^{2}, \\
& \gamma_{r}^{*}=-2+0.384616 \epsilon+0.117218 \epsilon^{2}, \\
& \gamma_{T}^{*}=-0.025534 \epsilon^{2}, \\
& \gamma_{\phi}{ }^{*}=-0.051068 \epsilon^{2} .
\end{aligned}
$$

We now solve the $R G$ equation (22) using the method of characteristics (see, for instance, Ref. [2]). To this end, we define $\mu^{\prime}(\sigma)=\mu \sigma$, where $\sigma$ is a dimensionless real parameter, and introduce the running parameters $s_{i}^{\prime}(\sigma)$ and the couplings $\lambda_{i}^{\prime}(\sigma)$ which respect following relations,

$$
\begin{gathered}
\sigma \frac{d s_{i}^{\prime}(\sigma)}{d \sigma}=s_{i}^{\prime}(\sigma) \gamma_{s_{i}}(\sigma), s^{\prime}(1)=s_{i}, \\
\sigma \frac{d \lambda_{i}^{\prime}(\sigma)}{d \sigma}=\lambda^{\prime}(\sigma) \beta_{i}(\sigma), \lambda^{\prime}(1)=\lambda_{i} .
\end{gathered}
$$

The $R G$ equation 22 together with the above relations yields,

$$
\begin{aligned}
& \Gamma_{\widetilde{n}, n}\left(q_{i}, s_{i}, \lambda_{i}, \mu\right)= \\
& \exp \left(\int_{1}^{\sigma} \frac{d \sigma^{\prime}}{\sigma^{\prime}} \frac{n}{2} \gamma_{\phi}(\sigma)\right) \Gamma_{\widetilde{n}, n}\left(q_{i}, s_{i}^{\prime}(\sigma), \lambda_{i}^{\prime}(\sigma), \mu \sigma\right) .
\end{aligned}
$$

At the fixed points, the solution to Eq. (28) gives simple power-law behavior, and at the $N E$ fixed point we obtain,

$$
s_{i}^{\prime}(\sigma) \approx s_{i} \sigma^{\gamma_{s_{i}}^{*}}
$$

Using the above result in Eq. (30), we obtain the critical scaling form of the vertex functions at the $N E$ fixed point,

$$
\Gamma_{\widetilde{n}, n}\left(q_{i}, s_{i}, \mu\right)=\sigma^{\frac{n}{2} \gamma_{\phi}^{*}} \Gamma_{\widetilde{n}, n}\left(q_{i}, s_{i} \sigma_{s_{i}}^{\gamma_{i}}, \mu \sigma\right),
$$

where we have not shown the arguments of $\Gamma$ which are not affected by rescaling. In the limit $r \rightarrow 0$, the parameter $\sigma$ scales as $\sigma \propto r^{-1 / \gamma_{r}^{*}}$, as can be seen from Eq. (31).

From Eq. (32) the scaling forms of the dynamic structure factor $S(\boldsymbol{q}, t)=\int_{q_{0}} e^{-i q_{0} t} \Gamma_{2,0}(q) /\left|\Gamma_{1,1}(q)\right|^{2}$ and the dynamic susceptibility $\chi(q)=1 / \Gamma_{1,1}(q)$ follow as

$$
\begin{aligned}
S\left(\boldsymbol{q}_{\perp}, q_{\|}, t, r\right)= & \sigma^{-2+\gamma_{T}^{*}-\gamma_{\phi}^{*}-\gamma_{D}^{*}} \\
& S\left(\frac{\boldsymbol{q}_{\perp}}{\sigma}, \frac{q_{\|}}{\sigma^{1-\gamma_{\rho}^{*} / 2}}, t \sigma^{2+\gamma_{D}^{*}}, \frac{r}{\sigma^{-\gamma_{r}^{*}}}\right), \\
\chi\left(\boldsymbol{q}_{\perp}, q_{\|}, t, r\right)= & \sigma^{-2-\gamma_{\phi}^{*} / 2-\gamma_{D}^{*}} \\
& \chi\left(\frac{\boldsymbol{q}_{\perp}}{\sigma}, \frac{q_{\|}}{\sigma^{1-\gamma_{\rho}^{*} / 2}}, t \sigma^{2+\gamma_{D}^{*}}, \frac{r}{\sigma^{-\gamma_{r}^{*}}}\right) .
\end{aligned}
$$

Comparing equations (33) and (34) with the standard 
scaling forms $[\underline{5}$,

$$
\begin{aligned}
& S\left(\boldsymbol{q}_{\perp}, q_{\|}, t, r\right)=\sigma^{-2+\eta} S\left(\frac{\boldsymbol{q}_{\perp}}{\sigma}, \frac{q_{\|}}{\sigma^{1+\Delta}}, t \sigma^{z}, \frac{r}{\sigma^{1 / \nu}}\right), \quad(35) \\
& \chi\left(\boldsymbol{q}_{\perp}, q_{\|}, t, r\right)=\sigma^{-z+\widetilde{\eta} / 2+\eta / 2} \chi\left(\frac{\boldsymbol{q}_{\perp}}{\sigma}, \frac{q_{\|}}{\sigma^{1+\Delta}}, \frac{q_{0}}{\sigma^{z}}, \frac{r}{\sigma^{1 / \nu}}\right),
\end{aligned}
$$

we obtain the exponents

$$
\begin{aligned}
\eta & =\gamma_{T}^{*}-\gamma_{D}^{*}-\gamma_{\phi}^{*}=0.0419341 \epsilon^{2}, \\
\Delta & =-\gamma_{\rho}^{*} / 2=0.1538455 \epsilon-0.00682625 \epsilon^{2}, \\
z & =2+\gamma_{D}^{*}=2-0.0164001 \epsilon^{2}, \\
\nu & =-1 / \gamma_{r}^{*}=0.5+0.192308 \epsilon+0.0955914 \epsilon^{2}, \\
\widetilde{\eta} & =\gamma_{D}^{*}-\gamma_{T}^{*}=0.0091339 \epsilon^{2},
\end{aligned}
$$

which are correct to second order in $\epsilon$.

The other standard anisotropy exponents 5 can be written in terms of the above five exponents. The transverse dynamic exponent $z_{\perp}=z$ and the longitudinal dynamic exponent $z_{\|}=z /(1+\Delta)$. Now, from Eq. (37), it follows that $z_{\|}<z_{\perp}$ which implies that the longitudinal fluctuations decay faster than the transverse fluctuations. The transverse correlation length exponent $\nu_{\perp}=\nu$, while the longitudinal correlation length exponent $\nu_{\|}=\nu(1+\Delta)$. As the strong anisotropy exponent $\Delta>0, \nu_{\|}>\nu_{\perp}$. This implies that the longitudinal correlation length diverges faster than the transverse correlation length as the temperature approaches the critical value.

There are four $\eta$-like exponents, two in the momentum space, and two in the real space. The two momentum space $\eta$-like exponents, $\eta_{\perp}^{M S}=\eta$ and $\eta_{\|}^{M S}=(\eta+$ $2 \Delta) /(1+\Delta)$, determine the anisotropic power-law behavior of the dynamic structure factor in momentum space and the two real space $\eta$-like exponents, $\eta_{\perp}^{R S}=\eta+\Delta$ and $\eta_{\|}^{R S}=(\eta-\Delta) /(1+\Delta)$, determine the anisotropic power-law behavior of the dynamic structure factor in real space. The relations obtained above are the same as the standard scaling relations observed in models that exhibit strong anisotropy [5].

The transverse and the longitudnal susceptibilities scale as $\chi_{\perp} \sim r^{-\gamma_{\perp}}$ and $\chi_{\|} \sim r^{-\gamma_{\|}}$, where $\chi_{\perp} \equiv \chi\left(\boldsymbol{q}_{\perp} \rightarrow\right.$ $\left.0, q_{\|}=0\right)$ and $\chi_{\|} \equiv \chi\left(\boldsymbol{q}_{\perp}=0, q_{\|} \rightarrow 0\right)$. From Eq. (36) we obtain

$$
\gamma_{\perp}=\gamma_{\|}=\nu(z-\widetilde{\eta} / 2-\eta / 2) .
$$

As opposed to the strongly anisotropic models that follow conserving dynamics, the susceptibility exponents $\gamma_{\perp}$ and $\gamma_{\|}$are equal [5].

To summarize, we studied the critical scaling behavior of the NSAPS three-vector model, which belongs to a new genuine non-equilibrium universality class. We obtained the critical exponents, which characterize the anisotropic power-law behavior of the dynamic structure factor and the dynamic susceptibility, to two-loop order. Among them is the important strong anisotropy exponent $\Delta$ that captures the effects of the spatially biased drive. We briefly mentioned the similarities and the dissimilarity in the critical behavior of the model to that of strongly anisotropic models that follow conserving dynamics.

\section{ACKNOWLEDGMENT}

I extend my sincere gratitude to Sreedhar B. Dutta, who suggested this problem and has spent hours engaging in fruitful discussions during the progress of the work and the preparation of the manuscript.

\section{Appendix A: Generating and evaluating Feynman diagrams: an example}

To obtain the Feynman diagrams that contribute to a vertex function, we first draw all the relevant topologies. For instance, to obtain the two-loop Feynman diagrams contributing to $\Gamma_{1,1}^{11}$, we draw all the distinct irreducible two-loop topologies with two external legs that can be constructed with three-point and four-point vertices, as shown in Fig. 2 Any two-loop contribution to $\Gamma_{1,1}^{11}$ must be topologically similar to one of these diagrams. Now, we use the building blocks shown in Fig. 1 to construct all the possible realizations of these topologies. This leads to the Feynman diagrams shown in Fig. 3.

Each of the diagrams in Fig. 3 corresponds to an expression that is of the form given in Eq. 16). For instance, diagram 22 corresponds to

$$
\mathcal{I}_{22}=12 T^{2} u_{0} e_{p}^{2} \int_{q_{1}, q_{2}}\left(q_{1 \|}+q_{2 \|}\right) q_{2 \|} C_{0}\left(q_{1}\right) C_{0}\left(q_{1}+q_{2}\right) G_{0}\left(-q_{1}-q_{2}\right) G_{0}\left(-q_{2}\right) .
$$

All the above steps were implemented in Mathematica [28] with the help of FeynCalc [26, 27] and FeynArts 25].

We can now proceed to extract the divergences. For instance, setting the external momenta to zero we obtain the quadratically divergent part which renormalizes the mass parameter $r$. The above integral does not depend on the external momenta and remains unchanged. Integrating out $q_{10}$ and $q_{20}$ from the above integral and making the 


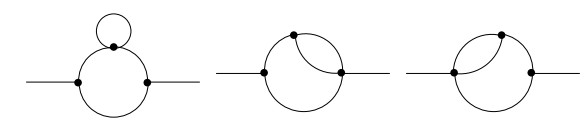

$\mathrm{T} 1$

$\mathrm{T} 2$

T3
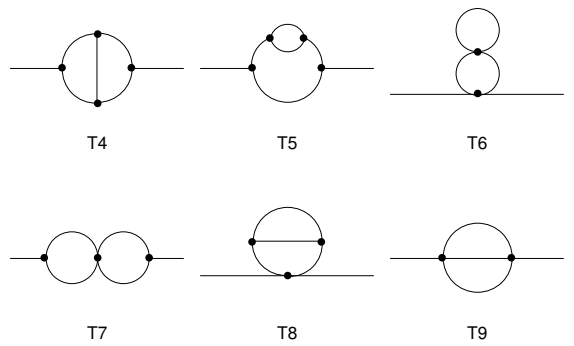

FIG. 2. All possible irreducible two-loop topologies with two external lines constructible with three-point and four-point vertices.
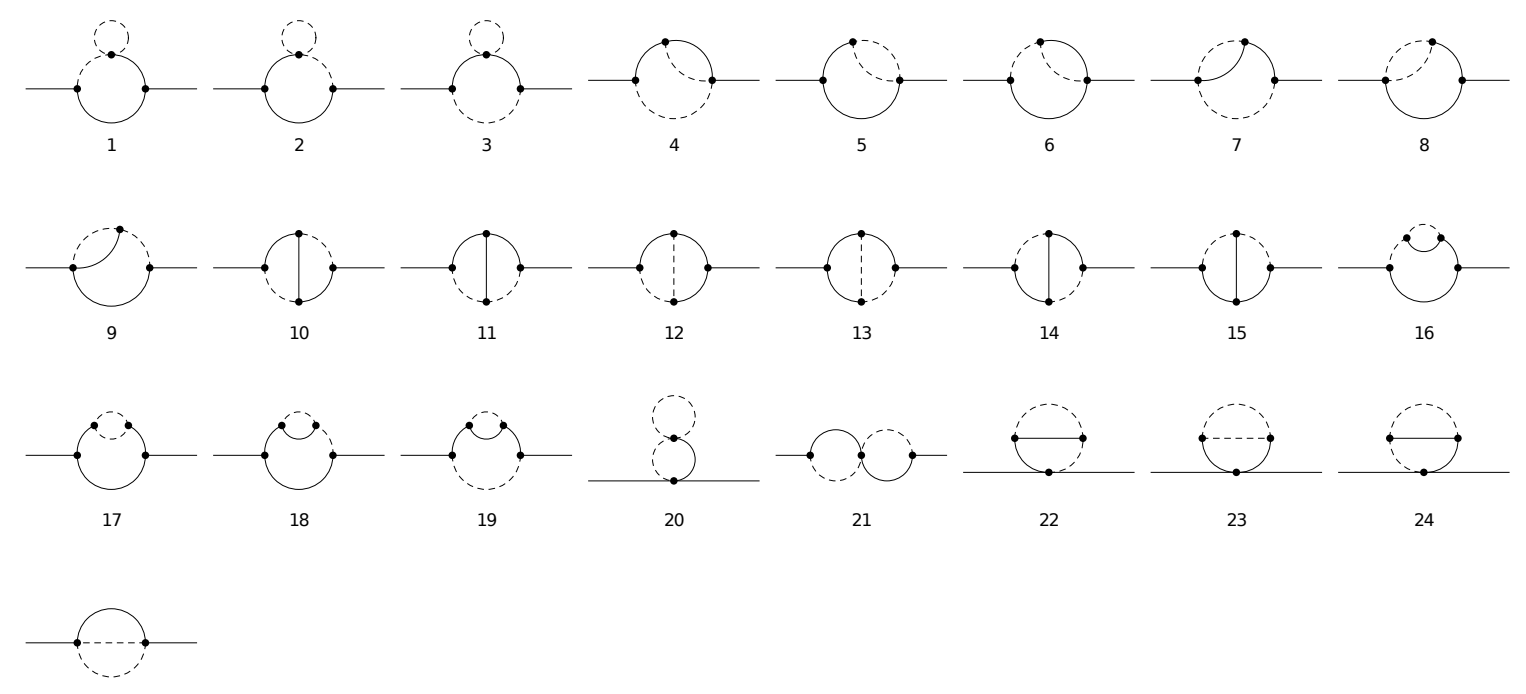

25

FIG. 3. Two-loop Feynman diagrams contributing to $\Gamma_{1,1}^{11}(q)$.

transformation, $\left\{\boldsymbol{q}_{i \perp}, \sqrt{\rho} q_{i \|}\right\} \rightarrow\left\{\sqrt{r} \boldsymbol{q}_{i \perp}, \sqrt{r} q_{i \|}\right\}$ we obtain,

$$
\mathcal{I}_{22}=\frac{3}{2} \frac{T^{2} u_{0} e_{p}^{2}}{D^{4} \rho^{2}} r^{d-3} \int_{\boldsymbol{q}_{1}, \boldsymbol{q}_{2}} \frac{\left(q_{1 \|}+q_{2 \|}\right) q_{2 \|}}{N\left(\boldsymbol{q}_{1}\right) N\left(\boldsymbol{q}_{1}+\boldsymbol{q}_{2}\right)^{2}\left(N\left(\boldsymbol{q}_{1}\right)+N\left(\boldsymbol{q}_{2}\right)+N\left(\boldsymbol{q}_{1}+\boldsymbol{q}_{2}\right)\right)}
$$

where, $N(\boldsymbol{q})=\boldsymbol{q}^{2}+1$. The UV-divergent parts of the above integral are expanded in powers of $\frac{1}{\epsilon}$ by employing dimensional regularization scheme with the help of the package SecDec 29.31.

$$
\widehat{\mathcal{D}}\left(\int_{\boldsymbol{q}_{1}, \boldsymbol{q}_{2}} \frac{q_{1 \|} q_{2 \|}}{N\left(\boldsymbol{q}_{1}\right) N\left(\boldsymbol{q}_{1}+\boldsymbol{q}_{2}\right)^{2}\left(N\left(\boldsymbol{q}_{1}\right)+N\left(\boldsymbol{q}_{2}\right)+N\left(\boldsymbol{q}_{1}+\boldsymbol{q}_{2}\right)\right)}\right)=\frac{1}{256 \pi^{4}}\left(\frac{0.125}{\epsilon^{2}}-\frac{0.563592}{\epsilon}\right)
$$

and

$$
\widehat{\mathcal{D}}\left(\int_{\boldsymbol{q}_{1}, \boldsymbol{q}_{2}} \frac{q_{2}^{2} \|}{N\left(\boldsymbol{q}_{1}\right) N\left(\boldsymbol{q}_{1}+\boldsymbol{q}_{2}\right)^{2}\left(N\left(\boldsymbol{q}_{1}\right)+N\left(\boldsymbol{q}_{2}\right)+N\left(\boldsymbol{q}_{1}+\boldsymbol{q}_{2}\right)\right)}\right)=\frac{1}{256 \pi^{4}}\left(-\frac{0.75}{\epsilon^{2}}+\frac{0.290792}{\epsilon}\right) .
$$


Eq. (A1) together with Eq. A2 gives the UV-divergent parts of diagram 22,

$$
\widehat{\mathcal{D}}\left(\mathcal{I}_{22}\right)=-\frac{3}{512 \pi^{4}} \frac{T^{2} u_{0} e_{p}^{2}}{D^{4} \rho^{2}} r^{d-3}\left(\frac{0.625}{\epsilon^{2}}+\frac{0.2728}{\epsilon}\right) .
$$

Appendix B: $\Gamma_{1,1}^{11}(0)$

Table $\mathrm{I}$ shows the only one-loop diagram contributing to $\Gamma_{1,1}^{11}(0)$ and its divergent contribution.

\begin{tabular}{|cc|}
\hline Diagrams & Divergence in $\epsilon$-expansion \\
\hline & $D r^{1-\epsilon / 2}\left(\frac{g_{0}}{2}+g_{1}\right) \frac{1}{\epsilon}$ \\
\hline & \\
\hline
\end{tabular}

The divergent parts of the two-loop diagrams contributing to $\Gamma_{1,1}^{11}(0)$ have the general form,

$$
\widehat{\mathcal{D}}(\mathcal{I}(q=0))=r^{1-\epsilon} D \mathcal{A}\left(\frac{n}{\epsilon}+\frac{m}{\epsilon^{2}}\right)
$$

where, $\mathcal{I}$ is the integral that a diagram represents and $n$ and $m$ are real numbers. The factor $\mathcal{A}$ is a function of the modified couplings

$$
g_{0} \equiv \frac{T}{8 \pi^{2} D^{2} \rho^{1 / 2}} u_{0}, g_{1} \equiv \frac{T}{8 \pi^{2} D^{2} \rho^{1 / 2}} u_{1} \text { and } g_{2} \equiv \frac{T}{8 \pi^{2} D^{3} \rho^{3 / 2}} e_{p}^{2}
$$

\begin{tabular}{|c|c|c|c|}
\hline Diagram & $\mathcal{A}, n, m$ & Diagram & $\mathcal{A}, n, m$ \\
\hline & $g_{1} g_{2},-0.1364,-0.3125$ & & $\left(g_{0}+2 g_{1}\right) g_{2},-0.0341,-0.078125$ \\
\hline & $g_{1} g_{2},-0.133328,-0.125$ & & $\left(g_{0}+2 g_{1}\right) g_{2}, 0.0846443,0.0625$ \\
\hline & $g_{1} g_{2}, 0.269728,0.4375$ & & $\left(g_{0}+2 g_{1}\right) g_{2},-0.0341,-0.078125$ \\
\hline & $\left(g_{0}+2 g_{1}\right)^{2}, 0.0193039,-0.25$ & & $g_{0}^{2}+6 g_{1}^{2},-0.230696,-0.25$ \\
\hline
\end{tabular}

Table [I] shows the two-loop diagrams and their respective contributions.

TABLE II: Two-loop contributions to $\Gamma_{1,1}^{11}(0)$

Collecting the divergences from all the above diagrams and applying the renormalization condition (6) we obtain the renormalization constant $Z_{r}$. In terms of the dimensionless renormalized couplings $\lambda_{i}$, which are defined in Eq. (19), $Z_{r}$ can be written explicitly as

$$
\begin{aligned}
Z_{r}= & 1+\frac{1}{\epsilon}\left(\lambda_{1}+0.5 \lambda_{0}-0.125 \lambda_{0}^{2}-0.75 \lambda_{1}^{2}+0.0560803 \lambda_{0} \lambda_{2}+0.112161 \lambda_{1} \lambda_{2}\right)+\frac{1}{\epsilon^{2}}\left(0.5 \lambda_{0}^{2}+2.5 \lambda_{1}^{2}+\lambda_{0} \lambda_{1}\right. \\
& \left.+0.09375 \lambda_{0} \lambda_{2}+0.1875 \lambda_{1} \lambda_{2}\right)
\end{aligned}
$$


Appendix C: $\left.\frac{\partial}{\partial q_{\|}^{2}} \Gamma_{1,1}^{11}(q)\right|_{q=0}$

Table III shows the only one-loop diagram contributing to $\left.\frac{\partial}{\partial q_{\|}^{2}} \Gamma_{1,1}^{11}(q)\right|_{q=0}$ and its divergent contribution.

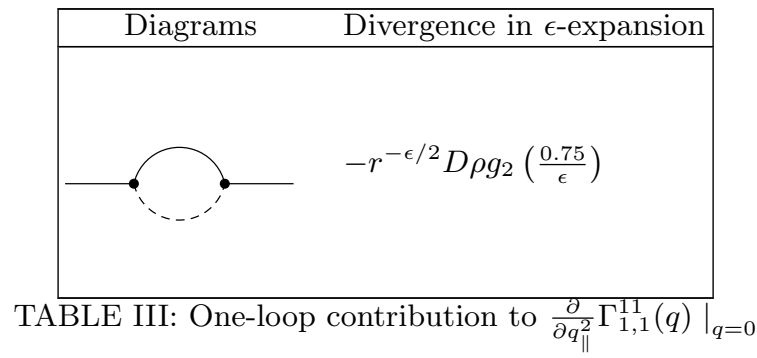

The divergent parts of the two-loop diagrams contributing to $\left.\frac{\partial}{\partial q_{\|}^{2}} \Gamma_{1,1}^{11}(q)\right|_{q=0}$ have the general form $r^{-\epsilon} D \rho \mathcal{A}\left(\frac{n}{\epsilon}+\frac{m}{\epsilon^{2}}\right)$. Table $\mathrm{IV}$ shows these diagrams and their respective divergences.

\begin{tabular}{|c|c|c|c|}
\hline Diagram & $\mathcal{A}, n, m$ & Diagram & $\mathcal{A}, n, m$ \\
\hline & $g_{0} g_{2}+2 g_{1} g_{2},-0.0520833,0$ & & $g_{2}^{2},-0.00835706,0.0234375$ \\
\hline & $g_{0} g_{2}+2 g_{1} g_{2},-0.09375,0$ & & $g_{2}^{2}, 0.0020853,-0.046875$ \\
\hline & $g_{0} g_{2}+2 g_{1} g_{2},-0.0416667,0$ & & $g_{2}^{2},-0.0180942,0.0234375$ \\
\hline & $g_{1} g_{2},-0.306357,0.375$ & & $g_{2}^{2}, 0.018626,-0.0117188$ \\
\hline & $g_{1} g_{2}, 0.143841,0$ & & $g_{2}^{2},-0.0401051,0.0234375$ \\
\hline & $g_{1} g_{2}, 0.0875039,-0.09375$ & & $g_{2}^{2},-0.0283968,0$ \\
\hline & $g_{1} g_{2},-0.216176,0.1875$ & & $g_{2}^{2},-0.0580853,0.0234375$ \\
\hline & $g_{1} g_{2},-0.0313306,0$ & & $g_{2}^{2},-0.0794423,0.09375$ \\
\hline
\end{tabular}




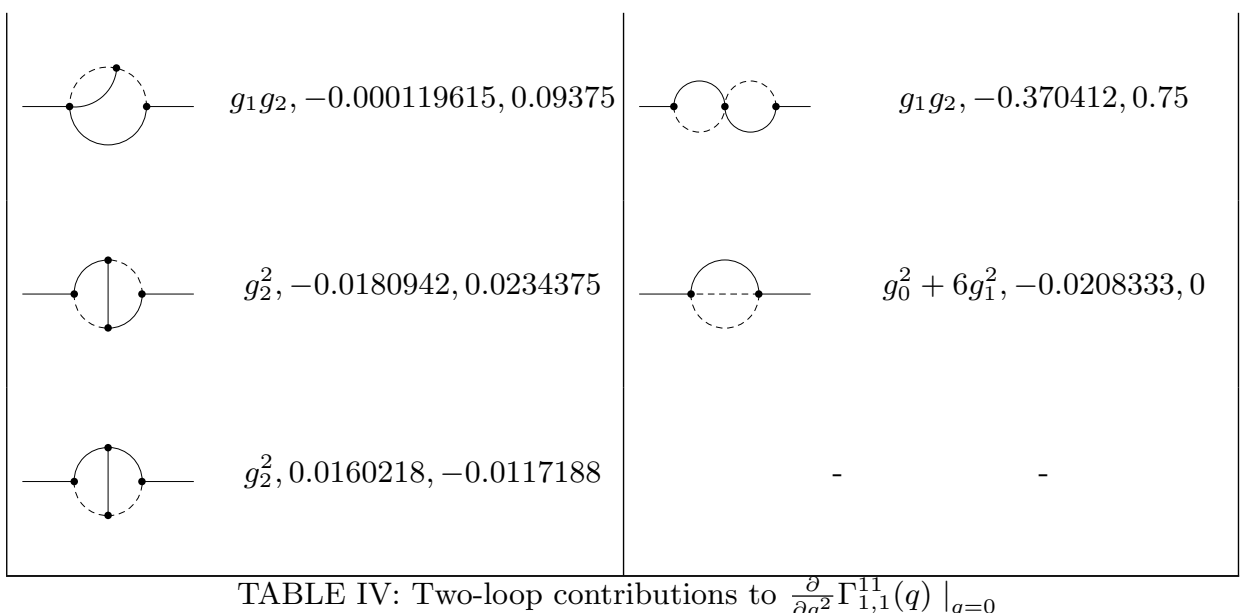

Collecting the divergences from all the above diagrams and applying the renormalization condition (7) yields,

$$
Z_{\rho}=1+\frac{1}{\epsilon}\left(-0.75 \lambda_{2}-0.0208333 \lambda_{0}^{2}-0.125 \lambda_{1}^{2}-0.156109 \lambda_{2}^{2}-0.154204 \lambda_{1} \lambda_{2}\right)+\frac{1}{\epsilon^{2}}\left(-0.140625 \lambda_{2}^{2}-1.3125 \lambda_{1} \lambda_{2}\right)
$$

Appendix D: $\left.\frac{\partial}{\partial i q_{0}} \Gamma_{1,1}(q)\right|_{q=0}$

There is no one-loop contribution to $\left.\frac{\partial}{\partial i q_{0}} \Gamma_{1,1}(q)\right|_{q=0}$. The divergent parts of the two-loop diagrams have the general form $r^{-\epsilon} \mathcal{A}\left(\frac{n}{\epsilon}+\frac{m}{\epsilon^{2}}\right)$. Table $\mathrm{V}$ shows these diagrams and their respective divergences.

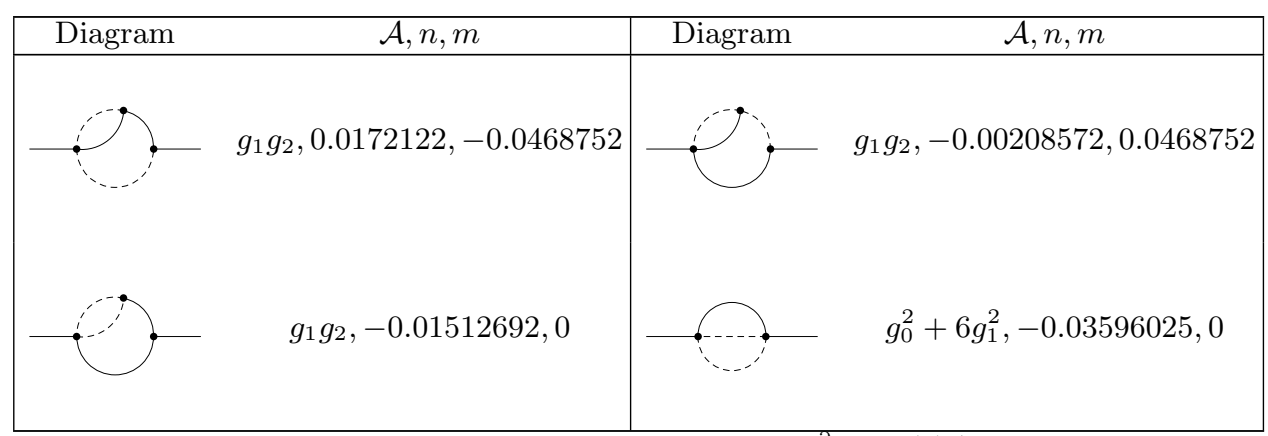

TABLE V: Two-loop contributions to $\left.\frac{\partial}{\partial i q_{0}} \Gamma_{1,1}(q)\right|_{q=0}$.

Collecting the divergences from all the above diagrams and applying the renormalization condition (8) yields,

$$
Z=1+\frac{1}{\epsilon}\left(-0.0359603 \lambda_{0}^{2}-0.215762 \lambda_{1}^{2}\right) .
$$

Appendix E: $\left.\frac{\partial}{\partial q_{\perp}^{2}} \Gamma_{1,1}^{11}(q)\right|_{q=0}$

There is no one-loop contribution to $\left.\frac{\partial}{\partial \boldsymbol{q}_{\perp}^{2}} \Gamma_{1,1}^{11}(q)\right|_{q=0}$. The divergent parts of the two-loop diagrams have the general form $r^{-\epsilon} D \mathcal{A}\left(\frac{n}{\epsilon}+\frac{m}{\epsilon^{2}}\right)$. Table $\mathrm{VI}$ shows these diagrams and their respective divergences. 


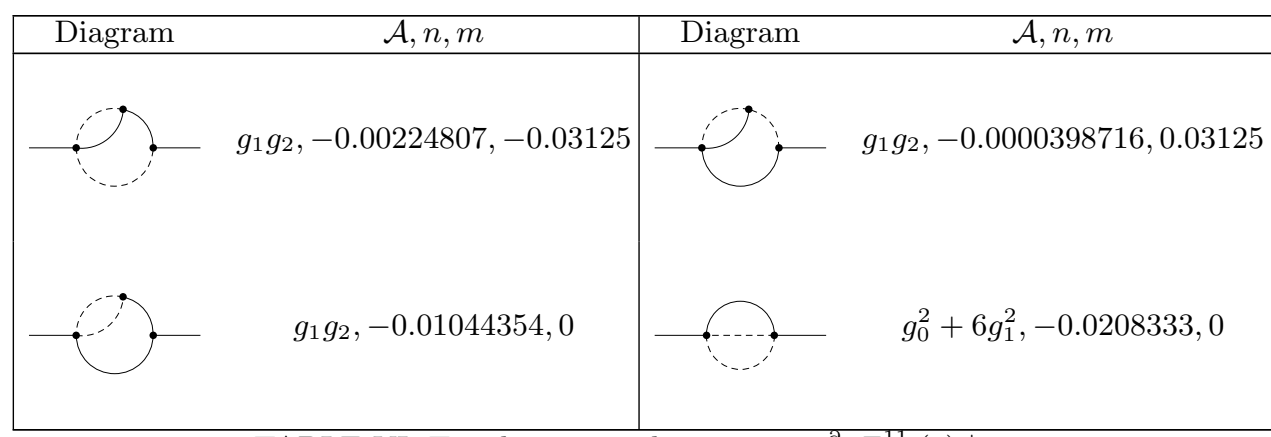

TABLE VI: Two-loop contributions to $\left.\frac{\partial}{\partial \boldsymbol{q}_{\perp}^{2}} \Gamma_{1,1}^{11}(q)\right|_{q=0}$.

Collecting the divergences from all the above diagrams and applying the renormalization condition (9) yields,

$$
Z_{D}=1+\frac{1}{\epsilon}\left(-0.0208333 \lambda_{0}^{2}-0.125 \lambda_{1}^{2}-0.0127315 \lambda_{1} \lambda_{2}\right)
$$

\section{Appendix F: $\left.\Gamma_{2,0}^{11}(q)\right|_{q=0}$}

There is no one-loop contribution to $\left.\Gamma_{2,0}^{11}(q)\right|_{q=0}$. The only two-loop contribution is given in Table VII.

\begin{tabular}{|cc|}
\hline Diagram & Divergence \\
\hline & \\
\hline
\end{tabular}

TABLE VII. Two-loop contribution to $\left.\Gamma_{2,0}^{11}(q)\right|_{q=0}$.

Collecting the divergence from the above diagram and applying the renormalization conditon 10 yields,

$$
Z_{T}=1+\frac{1}{\epsilon}\left(-0.0359603 \lambda_{0}^{2}-0.215762 \lambda_{1}^{2}\right)
$$

$$
\text { Appendix G: }\left.\frac{\partial}{\partial i q_{\|}} \Gamma_{1,2}^{123}\left(-q, \frac{q}{2}, \frac{q}{2}\right)\right|_{q=0}
$$

\begin{tabular}{|c|c|c|c|c|c|c|c|}
\hline Diagram & $\mathcal{A}, n$ & Diagram & $\mathcal{A}, n$ & Diagram & $\mathcal{A}, n$ & Diagram & $\mathcal{A}, n$ \\
\hline & $g_{2}, 0.125$ & &,-0 . & & $g_{1}, 1$ & & $g_{1}, 0.75$ \\
\hline
\end{tabular}

Table VIII shows the one-loop diagrams contributing to $\left.\frac{\partial}{\partial i q_{\|}} \Gamma^{123}{ }_{1,2}\left(-q, \frac{q}{2}, \frac{q}{2}\right)\right|_{q=0}$ and their respective divergent contributions. The divergent part of the one-loop diagrams have the general form $r^{-\epsilon / 2} e_{p} \mathcal{A}\left(\frac{n}{\epsilon}\right)$.

TABLE VIII: One-loop contributions to $\left.\frac{\partial}{\partial i q_{\|}} \Gamma_{1,2}^{123}\left(-q, \frac{q}{2}, \frac{q}{2}\right)\right|_{q=0}$. 
As the first and the second diagrams in Table VIII cancel each other there is no $g_{2}$ contribution to the renormalization constant $Z_{p}$ at the one-loop order.

Table $\operatorname{IX}$ shows the two-loop diagrams contributing to $\left.\frac{\partial}{\partial i q_{\|}} \Gamma_{1,2}^{123}\left(-q, \frac{q}{2}, \frac{q}{2}\right)\right|_{q=0}$.

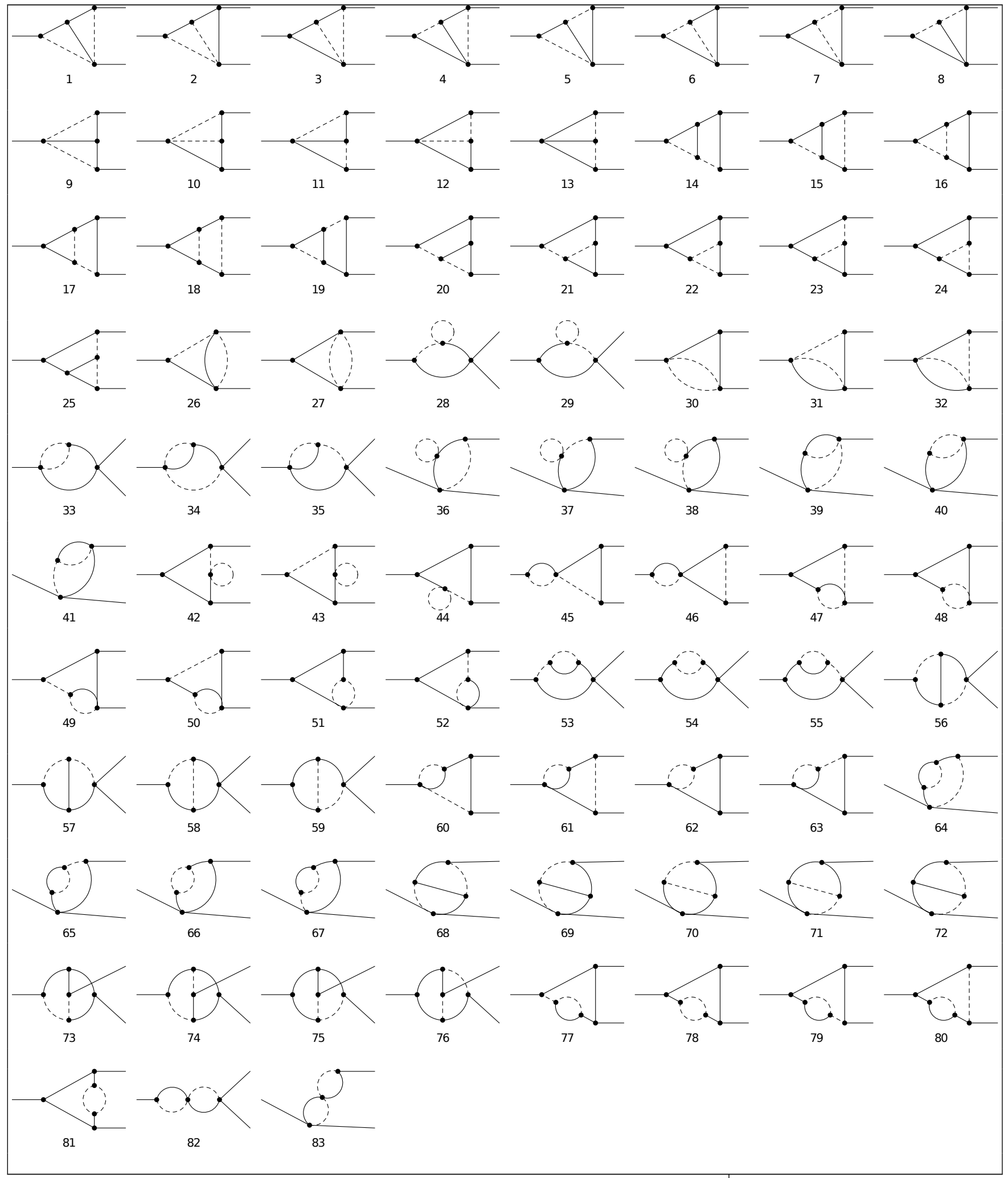

TABLE IX: Two-loop contributions to $\left.\frac{\partial}{\partial i q_{\|}} \Gamma_{1,2}^{123}\left(-q, \frac{q}{2}, \frac{q}{2}\right)\right|_{q=0}$ 
The divergent contributions of the above diagrams to $\left.\frac{\partial}{\partial i q_{\|}} \Gamma_{1,2}^{123}\left(-q, \frac{q}{2}, \frac{q}{2}\right)\right|_{q=0}$ have the general form, $r^{-\epsilon} e_{p} \mathcal{A}\left(\frac{n}{\epsilon}+\frac{m}{\epsilon^{2}}\right)$. The parameters $\mathcal{A}, n$ and $m$ for each of the above diagrams are listed against their respective diagram numbers in the table below.

\begin{tabular}{|c|c|c|c|}
\hline No. & $\mathcal{A}$ & $n$ & $m$ \\
\hline 1 & $\left(g_{0}+2 g_{1}\right) g_{2}$ & -0.00899006 & 0 \\
\hline 2 & $\left(g_{0}+2 g_{1}\right) g_{2}$ & -0.0132699 & 0 \\
\hline 3 & $\left(g_{0}+2 g_{1}\right) g_{2}$ & -0.0359603 & 0 \\
\hline 4 & $\left(g_{0}+2 g_{1}\right) g_{2}$ & 0.0201779 & -0.03125 \\
\hline 5 & $\left(g_{0}+2 g_{1}\right) g_{2}$ & 0.00427981 & 0 \\
\hline 6 & $\left(g_{0}+2 g_{1}\right) g_{2}$ & 0.0206083 & -0.03125 \\
\hline 7 & $\left(g_{0}+2 g_{1}\right) g_{2}$ & 0.0359603 & 0 \\
\hline 8 & $\left(g_{0}+2 g_{1}\right) g_{2}$ & -0.0540561 & 0.0625 \\
\hline 9 & $\left(g_{0}+2 g_{1}\right) g_{2}$ & 0.0125967 & -0.0117188 \\
\hline 10 & $\left(g_{0}+2 g_{1}\right) g_{2}$ & 0.000396799 & -0.0117188 \\
\hline 11 & $\left(g_{0}+2 g_{1}\right) g_{2}$ & -0.0297559 & 0.0117188 \\
\hline 12 & $\left(g_{0}+2 g_{1}\right) g_{2}$ & 0.00759028 & -0.0117188 \\
\hline 13 & $\left(g_{0}+2 g_{1}\right) g_{2}$ & -0.00502626 & 0.0234375 \\
\hline 14 & $g_{2}^{2}$ & 0.00328934 & -0.00390625 \\
\hline 15 & $g_{2}^{2}$ & -0.0160106 & 0.015625 \\
\hline 16 & $g_{2}^{2}$ & -0.00344199 & 0 \\
\hline 17 & $g_{2}^{2}$ & -0.000192796 & 0.0078125 \\
\hline 18 & $g_{2}^{2}$ & 0.00208366 & -0.0078125 \\
\hline 19 & $g_{2}^{2}$ & 0.0137743 & -0.0117188 \\
\hline 20 & $g_{2}^{2}$ & -0.0216832 & 0.015625 \\
\hline 21 & $g_{2}^{2}$ & 0.00586539 & -0.0078125 \\
\hline 22 & $g_{2}^{2}$ & 0.0123075 & 0 \\
\hline 23 & $g_{2}^{2}$ & -0.000657054 & 0.0078125 \\
\hline 24 & $g_{2}^{2}$ & -0.0160893 & 0 \\
\hline 25 & $g_{2}^{2}$ & 0.019079 & -0.015625 \\
\hline 26 & $g_{1}\left(2 g_{0}+3 g_{1}\right)$ & 0.127185 & -0.25 \\
\hline 27 & $g_{1}\left(2 g_{0}+3 g_{1}\right)$ & -0.107881 & 0 \\
\hline 28 & $g_{1}\left(g_{0}+2 g_{1}\right)$ & -0.00449503 & 0 \\
\hline 29 & $g_{1}\left(g_{0}+2 g_{1}\right)$ & -0.00449503 & 0 \\
\hline 30 & $g_{1}\left(2 g_{0}+3 g_{1}\right)$ & -0.0492301 & 0 \\
\hline 31 & $g_{1}\left(2 g_{0}+3 g_{1}\right)$ & 0.089859 & -0.09375 \\
\hline 32 & $g_{1}\left(2 g_{0}+3 g_{1}\right)$ & -0.0398513 & -0.09375 \\
\hline 33 & $g_{1}^{2}$ & 0.0302539 & 0 \\
\hline 34 & $g_{1}^{2}$ & 0.209432 & -0.28125 \\
\hline 35 & $g_{1}^{2}$ & 0.00417054 & -0.09375 \\
\hline 36 & $g_{1}\left(g_{0}+2 g_{1}\right)$ & -0.0230808 & 0 \\
\hline 37 & $g_{1}\left(g_{0}+2 g_{1}\right)$ & 0.09375 & 0 \\
\hline 38 & $g_{1}\left(g_{0}+2 g_{1}\right)$ & 0.0520833 & 0 \\
\hline 39 & $g_{1}^{2}$ & 0.306357 & -0.375 \\
\hline 40 & $g_{1}^{2}$ & -0.143841 & 0 \\
\hline 41 & & -0.0875039 & 0.09375 \\
\hline 42 & $\left(g_{0}+2 g_{1}\right) g_{2}$ & -0.03125 & 0 \\
\hline
\end{tabular}

\begin{tabular}{|c|c|c|c|}
\hline 43 & $\left(g_{0}+2 g_{1}\right) g_{2}$ & 0.015625 & 0 \\
\hline 44 & $\left(g_{0}+2 g_{1}\right) g_{2}$ & 0.015625 & 0 \\
\hline 45 & $g_{1} g_{2}$ & 0.103402 & -0.125 \\
\hline 46 & $g_{1} g_{2}$ & -0.103402 & 0.125 \\
\hline 47 & $g_{1} g_{2}$ & -0.0901321 & 0.125 \\
\hline 48 & $g_{1} g_{2}$ & 0.0719206 & 0 \\
\hline 49 & $g_{1} g_{2}$ & 0.0875039 & -0.09375 \\
\hline 50 & $g_{1} g_{2}$ & 0.126092 & -0.125 \\
\hline 51 & $g_{1} g_{2}$ & -0.0719206 & 0 \\
\hline 52 & $g_{1} g_{2}$ & -0.0609641 & 0.09375 \\
\hline 53 & $g_{1} g_{2}$ & 0.0875039 & -0.09375 \\
\hline 54 & $g_{1} g_{2}$ & 0.0719206 & 0 \\
\hline 55 & $g_{1} g_{2}$ & 0.0609641 & -0.09375 \\
\hline 56 & $g_{1} g_{2}$ & 0.0201779 & -0.03125 \\
\hline 57 & $g_{1} g_{2}$ & 0.00690802 & -0.03125 \\
\hline 58 & $g_{1} g_{2}$ & -0.0132699 & 0 \\
\hline 59 & $g_{1} g_{2}$ & -0.0138161 & 0.0625 \\
\hline 60 & $g_{1} g_{2}$ & 0.0854314 & -0.0820313 \\
\hline 61 & $g_{1} g_{2}$ & -0.0333353 & 0.046875 \\
\hline 62 & $g_{1} g_{2}$ & -0.0121528 & 0 \\
\hline 63 & $g_{1} g_{2}$ & -0.0115466 & 0.0351563 \\
\hline 64 & $g_{1} g_{2}$ & 0.105484 & -0.09375 \\
\hline 65 & $g_{1} g_{2}$ & 0.00600193 & -0.0234375 \\
\hline 66 & $g_{1} g_{2}$ & 0.0283968 & 0 \\
\hline 67 & $g_{1} g_{2}$ & 0.0140635 & -0.0234375 \\
\hline 68 & $g_{1} g_{2}$ & 0.0361885 & -0.046875 \\
\hline 69 & $g_{1} g_{2}$ & -0.0160218 & 0.0117188 \\
\hline 70 & $g_{1} g_{2}$ & -0.00208526 & 0.046875 \\
\hline 71 & $g_{1} g_{2}$ & 0.0135654 & -0.0234375 \\
\hline 72 & $g_{1} g_{2}$ & -0.018626 & 0.0117187 \\
\hline 73 & $g_{2}^{2}$ & 0.00806154 & 0 \\
\hline 74 & $g_{2}^{2}$ & -0.00471026 & 0 \\
\hline 75 & $g_{2}^{2}$ & 0.00378175 & 0 \\
\hline 76 & $g_{2}^{2}$ & -0.00378175 & 0 \\
\hline 77 & $g_{2}^{2}$ & 0.0445327 & -0.0351563 \\
\hline 78 & $g_{2}^{2}$ & 0.0217619 & 0 \\
\hline 79 & $g_{2}^{2}$ & 0.0274811 & -0.0351563 \\
\hline 80 & $g_{2}^{2}$ & -0.0757955 & 0.0703125 \\
\hline 81 & $g_{2}^{2}$ & -0.0179801 & 0 \\
\hline 82 & $g_{1}^{2}$ & 0.577216 & -1 \\
\hline 83 & $g_{1}^{2}$ & 0.370412 & -0.75 \\
\hline
\end{tabular}

Collecting the divergences from all the above diagrams and applying the renormalization condition 11 yields,

$$
\begin{aligned}
Z_{p}= & 1+\frac{1}{\epsilon}\left(1.75 \lambda_{1}-1.20163 \lambda_{1}^{2}+0.00167566 \lambda_{2}^{2}-0.726138 \lambda_{0} \lambda_{1}+0.221394 \lambda_{1} \lambda_{2}-0.0454484 \lambda_{0} \lambda_{2}\right)+\frac{1}{\epsilon^{2}}\left(3.71875 \lambda_{1}^{2}\right. \\
& \left.+0.875 \lambda_{0} \lambda_{1}+0.328125 \lambda_{1} \lambda_{2}\right)
\end{aligned}
$$




\section{Appendix H: $\Gamma_{1,3}^{111}(0)$}

Table XI shows the one-loop diagrams contributing to $\Gamma_{1,3}^{1111}(0)$ and their respective divergent contributions. The divergent part of the one-loop diagrams have the general form $r^{-\epsilon / 2} u_{0} \mathcal{A}\left(\frac{n}{\epsilon}\right)$.

\begin{tabular}{|cc|cc|cc|}
\hline Diagram & $\mathcal{A}, n$ & Diagram & $\mathcal{A}, n$ & Diagram & $\mathcal{A}, n$ \\
\hline & $\frac{g_{1} g_{2}}{g_{0}}, 0.75$ & & & & \\
\hline
\end{tabular}

TABLE XI: One-loop contributions to $\Gamma_{1,3}^{111}(0)$.

As the first two diagrams in Table XI] cancel each other there is no $g_{2}$ contribution to the renormalization constant $Z_{0}$ at the one-loop order. Table XII shows the two-loop diagrams contributing to $\Gamma_{1,3}^{111}(0)$.

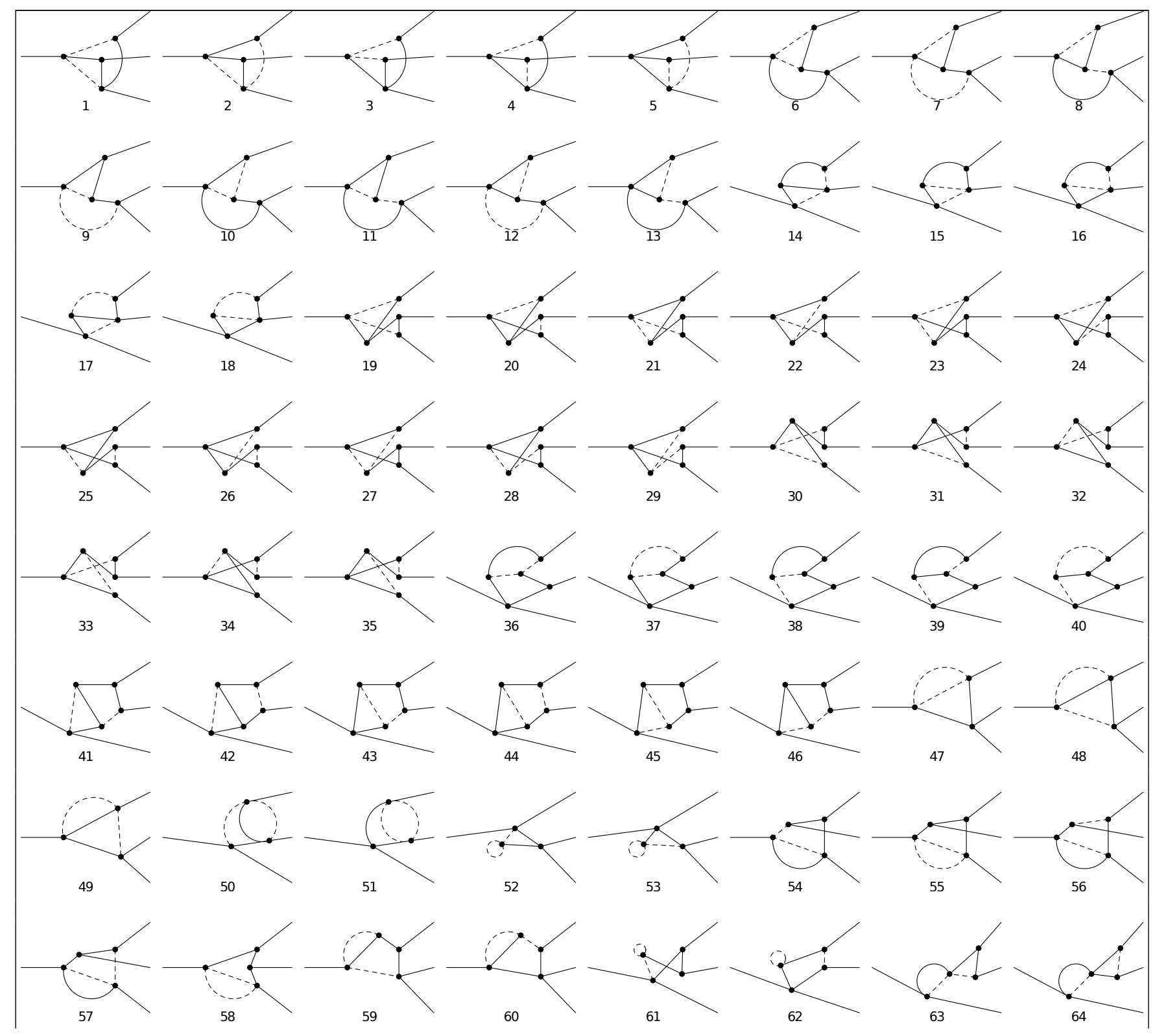




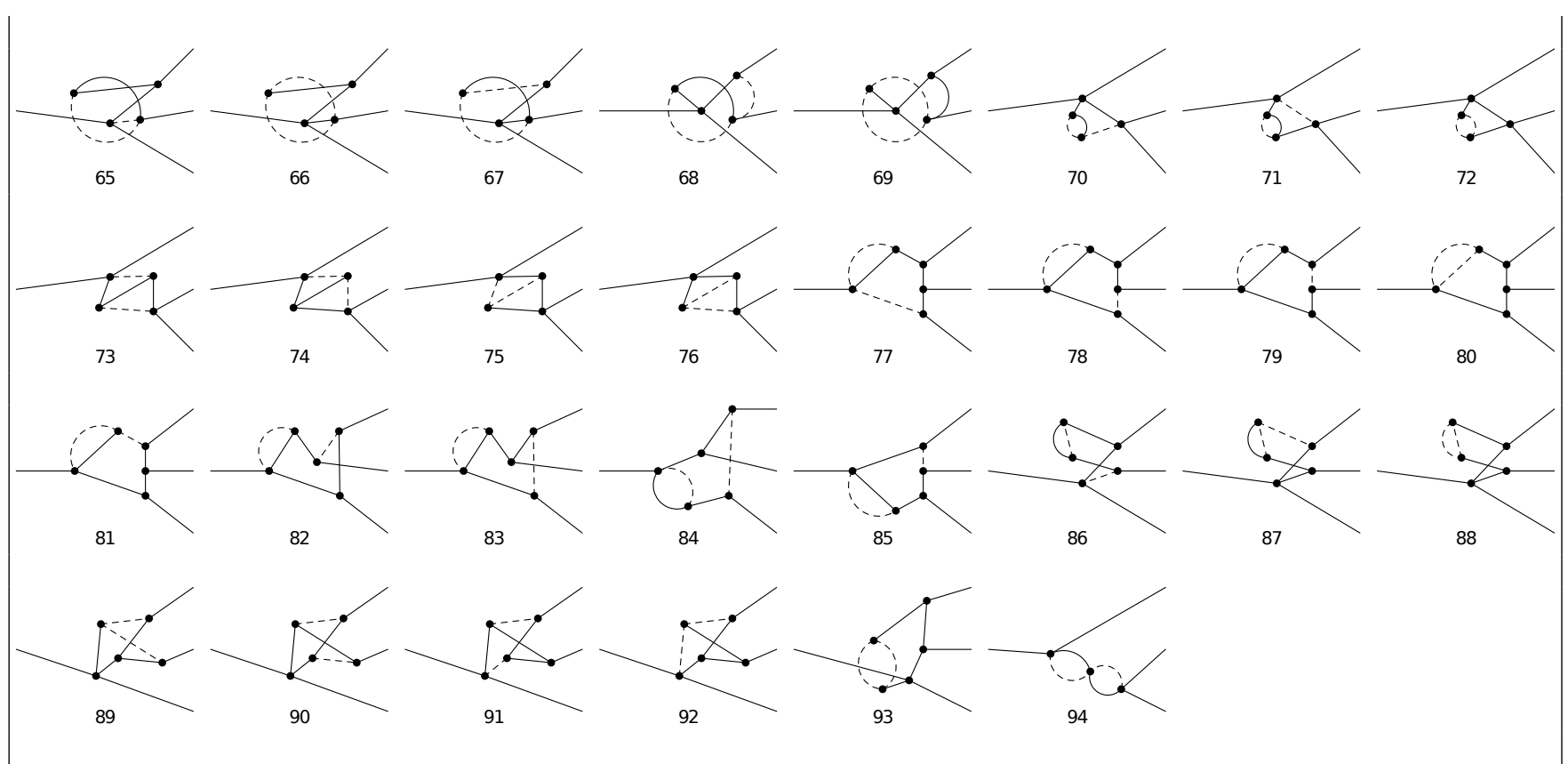

TABLE XII: Two-loop diagrams contributing to $\Gamma_{1,3}^{111}(0)$

The divergent contributions of the above diagrams to $\Gamma_{1,3}^{111}(0)$ have the general form $r^{-\epsilon} u_{0} \mathcal{A}\left(\frac{n}{\epsilon}+\frac{m}{\epsilon^{2}}\right)$. The parameters $\mathcal{A}, n$ and $m$ for each of the above diagrams are listed against their respective diagram numbers in the table below.

\begin{tabular}{|l|l|l|l|}
\hline No. & $\mathcal{A}$ & $n$ & $m$ \\
\hline 1 & $\frac{g_{1}^{2} g_{2}}{g_{0}}$ & -0.0796192 & 0 \\
2 & $\frac{g_{1}^{2} g_{2}}{g_{0}}$ & 0.170381 & 0 \\
3 & $\frac{g_{1}^{2} g_{2}}{g_{0}}$ & 0.0128394 & 0 \\
4 & $\frac{g_{1}^{2} g_{2}}{g_{0}}$ & -0.0539404 & 0 \\
5 & $\frac{g_{1}^{2} g_{2}}{g_{0}}$ & 0.0582202 & 0 \\
6 & $\frac{g_{1}\left(g_{0}+g_{1}\right) g_{2}}{g_{0}}$ & -0.0398096 & 0 \\
7 & $\frac{g_{1}\left(g_{0}+g_{1}\right) g_{2}}{g_{0}}$ & 0.181601 & -0.28125 \\
8 & $\frac{g_{1}\left(g_{0}+g_{1}\right) g_{2}}{g_{0}}$ & 0.0207241 & -0.09375 \\
9 & $\frac{g_{1}\left(g_{0}+g_{1}\right) g_{2}}{g_{0}}$ & 0.185475 & -0.28125 \\
10 & $\frac{g_{1}\left(g_{0}+g_{1}\right) g_{2}}{g_{0}}$ & 0.0851904 & 0 \\
11 & $\frac{g_{1}\left(g_{0}+g_{1}\right) g_{2}}{g_{0}}$ & -0.0289365 & -0.09375 \\
12 & $\frac{g_{1}\left(g_{0}+g_{1}\right) g_{2}}{g_{0}}$ & -0.486505 & 0.5625 \\
13 & $\frac{g_{1}\left(g_{0}+g_{1}\right) g_{2}}{g_{0}}$ & -0.0769779 & 0.1875 \\
14 & $\frac{g_{1}\left(g_{0}+g_{1}\right) g_{2}}{g_{0}}$ & -0.0539404 & 0 \\
15 & $\frac{g_{1}\left(g_{0}+g_{1}\right) g_{2}}{g_{0}}$ & -0.0796192 & 0 \\
16 & $\frac{g_{1}\left(g_{0}+g_{1}\right) g_{2}}{g_{0}}$ & -0.215762 & 0 \\
17 & $\frac{g_{1}\left(g_{0}+g_{1}\right) g_{2}}{g_{0}}$ & 0.0256788 & 0 \\
18 & $\frac{g_{1}\left(g_{0}+g_{1}\right) g_{2}}{g_{0}}$ & 0.215762 & 0 \\
19 & $\frac{g_{1} g_{2}^{2}}{g_{0}}$ & $\frac{g_{1} g_{2}^{2}}{g_{0}}$ & $\frac{g_{1} g_{2}^{2}}{g_{0}}$ \\
22 & $\frac{g_{1} g_{2}^{2}}{g_{0}}$ & -0459143 & -0.0390625 \\
20 & 0.0293269 & -0.0390625 \\
\hline
\end{tabular}

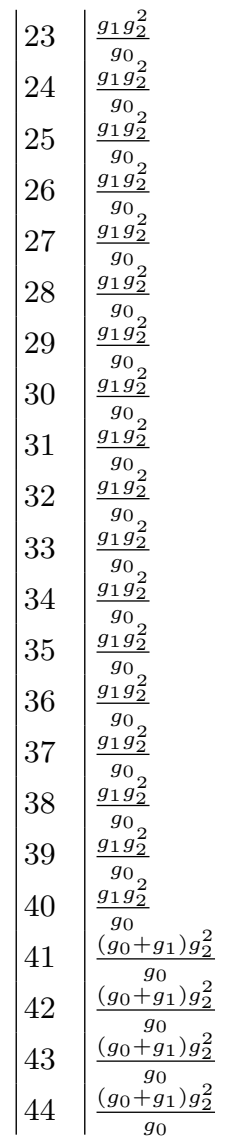

\begin{tabular}{l|l|}
-0.0206519 & 0 \\
0.0197361 & -0.0234375 \\
-0.00657054 & 0.078125 \\
0.19079 & -0.15625 \\
0.0522647 & 0 \\
-0.0415526 & -0.0234375 \\
-0.0468204 & 0.046875 \\
0.00918285 & -0.0078125 \\
-0.0160106 & 0.015625 \\
0.00586539 & -0.0078125 \\
-0.0216832 & 0.015625 \\
-0.00131411 & 0.015625 \\
0.038158 & -0.03125 \\
-0.0965356 & 0 \\
0.0738452 & 0 \\
0.0175962 & -0.0234375 \\
0.0275486 & -0.0234375 \\
-0.0650495 & 0.046875 \\
0.0275486 & -0.0234375 \\
-0.0480317 & 0.046875 \\
-0.00115712 & 0.046875 \\
0.0125021 & -0.046875
\end{tabular}




\begin{tabular}{|c|c|c|c|}
\hline 45 & $\frac{\left(g_{0}+g_{1}\right) g_{2}^{2}}{g_{0}}$ & -0.0206519 & 0 \\
\hline 4 & $\underline{\left(g_{0}+g_{1}\right) g_{2}^{2}}$ & 0.0197361 & -0.0234375 \\
\hline 47 & $\frac{g_{0}^{3}+2 g_{1}^{2} g_{0}+4 g_{1}^{3}}{g_{0}}$ & -0.107881 & 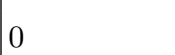 \\
\hline 4 & $\frac{g_{0}^{3}+2 g_{1}^{2} g_{0}+4 g_{1}^{3}}{a_{0}}$ & 0.381554 & -0.75 \\
\hline 49 & $\frac{g_{0}^{3}+2 g_{1}^{20} g_{0}+4 g_{1}^{3}}{a_{0}}$ & -0.0249845 & -0.375 \\
\hline 5 & $\frac{g_{0}^{3}+2 g_{1}^{g_{0}} g_{0}+4 g_{1}^{3}}{g_{0}}$ & 0.190777 & -0.375 \\
\hline & $\underline{g_{0}^{3}+2 g_{1}^{2} g_{0}+4 g_{1}^{3}}$ & -0.323643 & 0 \\
\hline & $\underline{g_{0}^{3}+2 g_{1} g_{0}^{2}+2 g_{1}^{2} g_{0}+4 g_{1}^{3}}$ & 0.1875 & 0 \\
\hline & $\underline{g_{0}^{3}+2 g_{1} g_{0}^{2}+2 g_{1}^{2} g_{0}+4 g_{1}^{3}}$ & 0.1875 & 0 \\
\hline & $\begin{array}{ll}g_{1}^{2} g_{2} & g_{0}\end{array}$ & & \\
\hline & $\frac{1192}{g_{0}}$ & 0.378277 & -0.375 \\
\hline 5 & $\frac{g_{1}^{2} g_{2}}{g_{0}}$ & -0.136142 & 0 \\
\hline 5 & $\frac{g_{1}^{2} g_{2}}{g_{0}}$ & -0.540793 & 0.75 \\
\hline 57 & $\frac{g_{1}^{2} g_{2}}{g_{0}}$ & 0.0375155 & -0.375 \\
\hline 58 & $\frac{g_{1}^{2} g_{2}}{g_{0}}$ & -0.0453808 & 0 \\
\hline 59 & $\frac{g_{1}^{2} g_{2}}{g_{0}}$ & 0.787535 & -0.84375 \\
\hline 60 & $\frac{g_{1}^{2} g_{2}}{g_{0}}$ & -0.0125116 & 0.28125 \\
\hline 61 & $\frac{g_{1}\left(g_{0}+2 g_{1}\right) g_{2}}{g_{0}}$ & 0.09375 & 0 \\
\hline 62 & $\frac{g_{1}\left(g_{0}+2 g_{1}\right) g_{2}}{g_{0}}$ & -0.09375 & 0 \\
\hline 63 & $\frac{g_{1}\left(2 g_{0}+g_{1}\right) g_{2}}{g_{0}}$ & 0.310206 & -0.375 \\
\hline 64 & $\frac{g_{1}\left(2 g_{0}+g_{1}\right) g_{2}}{g_{0}}$ & -0.310206 & 0.375 \\
\hline 65 & $\frac{g_{1}^{2} g_{2}}{g_{0}}$ & 0.756554 & -0.75 \\
\hline 66 & $\frac{g_{1}^{2} g_{2}}{g_{0}}$ & -0.431524 & 0 \\
\hline 67 & $\frac{g_{1}^{2} g_{2}}{g_{0}}$ & -0.365785 & 0.5625 \\
\hline 68 & $\frac{g_{1}^{2} g_{2}}{g_{0}}$ & -0.540793 & 0.75 \\
\hline 69 & $\frac{g_{1}^{2} g_{2}}{g_{0}}$ & 0.431524 & 0 \\
\hline
\end{tabular}

\begin{tabular}{|c|c|c|c|}
\hline 70 & $\frac{\left(g_{0}^{2}+2 g_{1}^{2}\right) g_{2}}{g_{0}}$ & 0.0914462 & -0.140625 \\
\hline 71 & $\frac{\left(g_{0}^{2}+2 g_{1}^{2}\right) g_{2}}{g_{0}}$ & 0.131256 & -0.140625 \\
\hline 72 & $\frac{\left(g_{0}^{2}+2 g_{1}^{2}\right) g_{2}}{g_{0}}$ & 0.107881 & 0 \\
\hline $7:$ & $\frac{g_{1}\left(2 g_{0}+g_{1}\right) g_{2}}{g_{0}}$ & 0.0605337 & -0.09375 \\
\hline 74 & $\frac{g_{1}\left(2 g_{0}+g_{1}\right) g_{2}}{g_{0}}$ & 0.0207241 & -0.09375 \\
\hline & $\frac{g_{1}\left(2 g_{0}+g_{1}\right) g_{2}}{g_{0}}$ & -0.0398096 & 0 \\
\hline 76 & $\frac{g_{1}\left(2 g_{0}+g_{1}\right) g_{2}}{g_{0}}$ & -0.0414484 & 0.1875 \\
\hline 77 & $\frac{g_{1} g_{2}^{2}}{g_{0}}$ & 0.267196 & -0.210938 \\
\hline 78 & $\frac{g_{1} g_{2}^{2}}{g_{0}}$ & -0.227387 & 0.210938 \\
\hline 79 & $\frac{g_{1} g_{2}^{2}}{g_{0}}$ & 0.0549622 & -0.0703125 \\
\hline 80 & $\frac{g_{1} g_{2}^{2}}{g_{0}}$ & -0.0210752 & 0 \\
\hline 81 & $\frac{g_{1} g_{2}^{2}}{g_{0}}$ & -0.00920429 & 0.0703125 \\
\hline 82 & $\frac{g_{1} g_{2}^{2}}{g_{0}}$ & 0.24733 & -0.316406 \\
\hline 83 & $\frac{g_{1} g_{2}^{2}}{g_{0}}$ & -0.151591 & 0.140625 \\
\hline 84 & $\frac{g_{1} g_{2}^{2}}{g_{0}}$ & 0.0274811 & -0.0351563 \\
\hline 85 & $\frac{g_{1} g_{2}^{2}}{g_{0}}$ & -0.0757955 & 0.0703125 \\
\hline 86 & $\frac{g_{1} g_{2}^{2}}{g_{0}}$ & 0.0890654 & -0.0703125 \\
\hline 87 & $\frac{g_{1} g_{2}^{2}}{g_{0}}$ & -0.227387 & 0.210938 \\
\hline 88 & $\frac{g_{1} g_{2}^{2}}{g_{0}}$ & -0.107881 & 0 \\
\hline 89 & $g_{2}^{20}$ & 0.0113452 & 0 \\
\hline 90 & $g_{2}^{2}$ & -0.0113452 & 0 \\
\hline 91 & $g_{2}^{2}$ & -0.0141308 & 0 \\
\hline 92 & $g_{2}^{2}$ & 0.0241846 & 0 \\
\hline 93 & $\frac{g_{1} g_{2}^{2}}{g_{0}}$ & 0.130571 & 0 \\
\hline 94 & $\frac{g_{0}^{3}+6 g_{1}^{2} g_{0}+2 g_{1}^{3}}{g_{0}}$ & 0.432912 & -0.75 \\
\hline
\end{tabular}

Collecting the divergences from all the above diagrams and applying the renormalization condition 12 yields,

$$
\begin{aligned}
Z_{0}= & +\frac{1}{\epsilon}\left(1.5 \lambda_{0}-0.75 \lambda_{0}^{2}-1.5 \lambda_{1}^{2}-0.26712 \lambda_{1} \lambda_{2}+0.168241 \lambda_{0} \lambda_{2}-\frac{3 \lambda_{1}^{3}}{\lambda_{0}}+\frac{0.49572 \lambda_{2} \lambda_{1}^{2}}{\lambda_{0}}+\frac{3 \lambda_{1}^{2}}{\lambda_{0}}-\frac{0.00819685 \lambda_{2}^{2} \lambda_{1}}{\lambda_{0}}\right) \\
& +\frac{1}{\epsilon^{2}}\left(2.25 \lambda_{0}^{2}+7.5 \lambda_{1}^{2}-+0.28125 \lambda_{0} \lambda_{2}+\frac{7.5 \lambda_{1}^{3}}{\lambda_{0}}+\frac{0.5625 \lambda_{2} \lambda_{1}^{2}}{\lambda_{0}}\right)
\end{aligned}
$$

\section{Appendix I: $\Gamma_{1,3}^{1122}(0)$}

Table XIV shows the one-loop diagrams contributing to $\Gamma_{1,3}^{1122}(0)$ and their respective divergent contributions. The divergent part of the one-loop diagrams have the general form $r^{-\epsilon / 2} u_{1} \mathcal{A}\left(\frac{n}{\epsilon}\right)$.

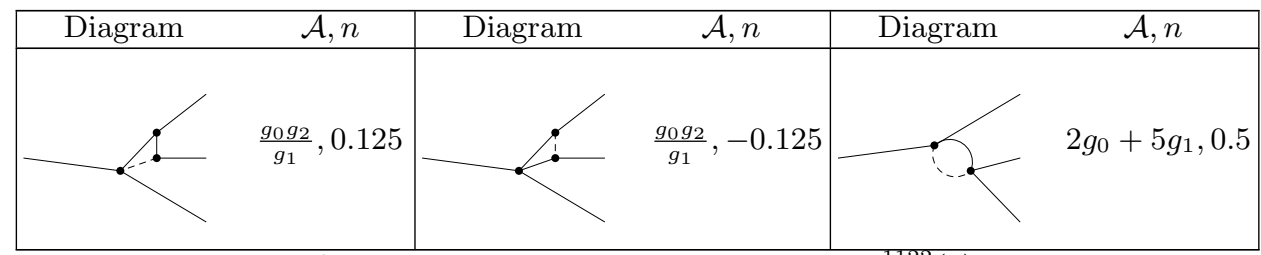

TABLE XIV: One-loop contributions to $\Gamma_{1,3}^{1122}(0)$. 
As the first two diagrams in Table XIV cancel each other there is no $g_{2}$ contribution to the renormalization constant $Z_{1}$ at the one-loop order. Table XV shows the two-loop diagrams contributing to $\Gamma_{1,3}^{1122}(0)$.

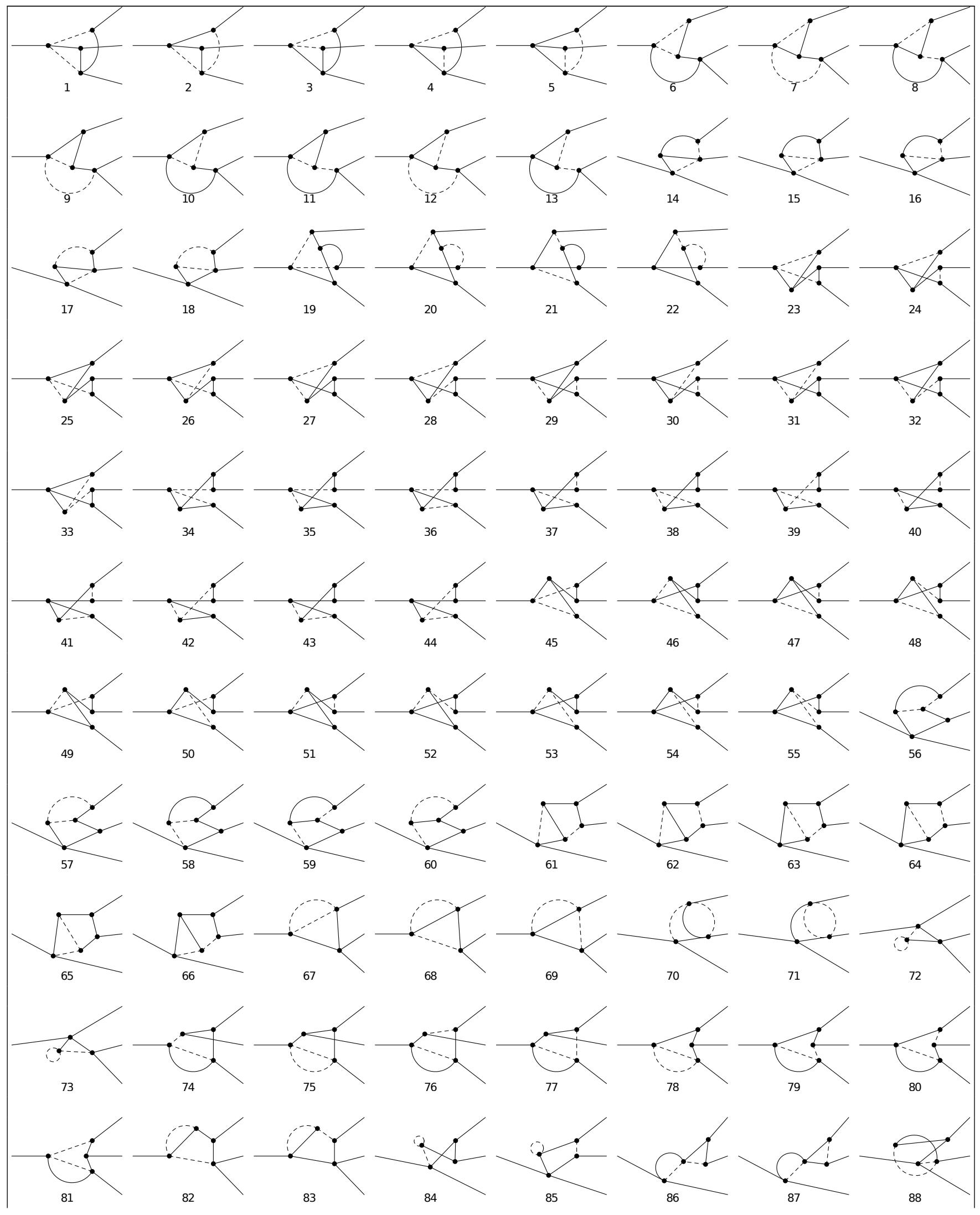




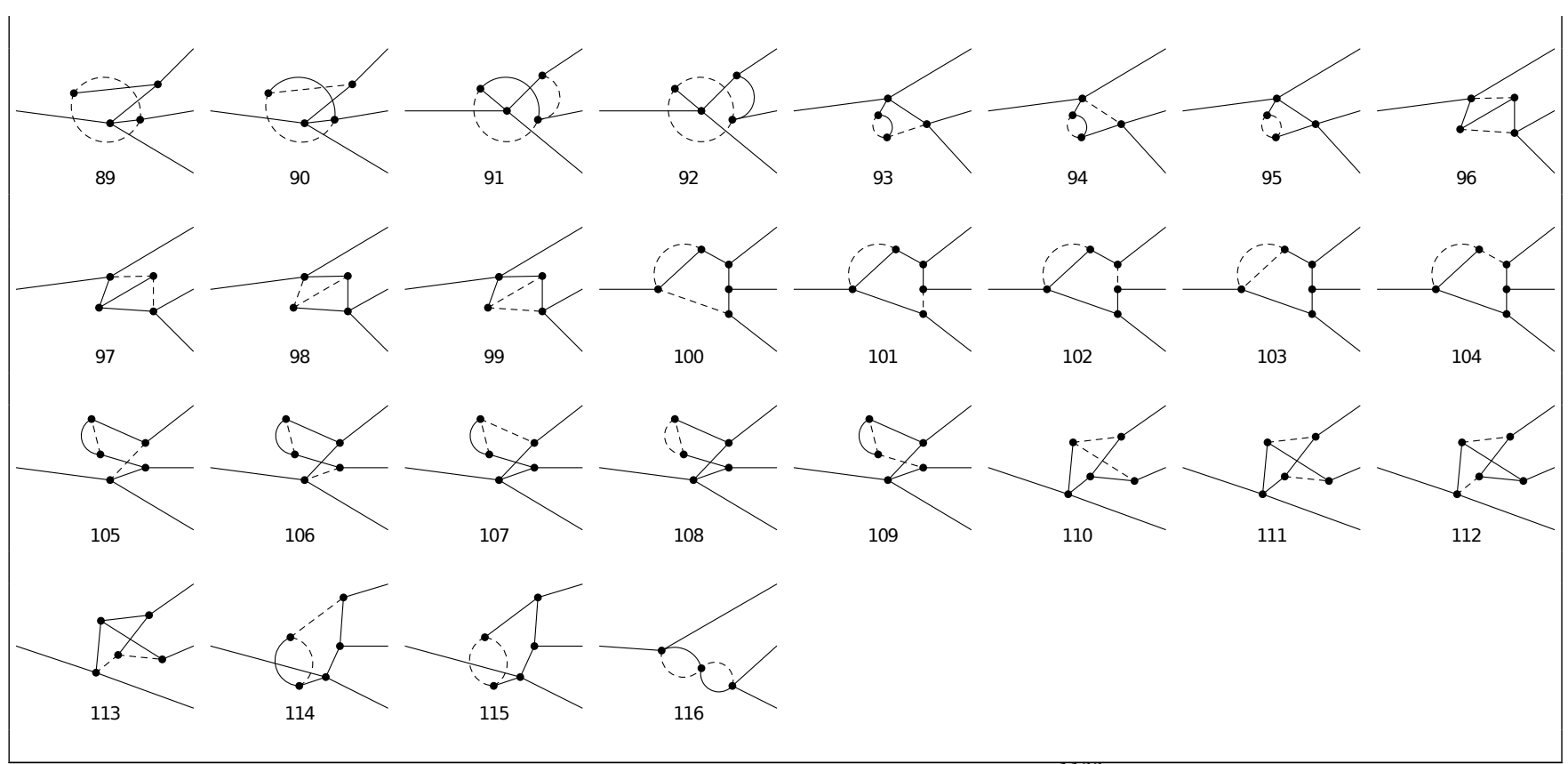

TABLE XV: Two-loop diagrams contributing to $\Gamma_{1,3}^{1122}(0)$

The divergent contributions of the above diagrams to $\Gamma_{1,3}^{1122}(0)$ have the general form $r^{-\epsilon} u_{1} \mathcal{A}\left(\frac{n}{\epsilon}+\frac{m}{\epsilon^{2}}\right)$. The parameters $\mathcal{A}, n$, and $m$ for each of the above diagrams are listed against their respective diagram numbers in the table below.

\begin{tabular}{|l|l|l|l|}
\hline No. & $\mathcal{A}$ & $n$ & $m$ \\
\hline 1 & $\left(g_{0}+4 g_{1}\right) g_{2}$ & -0.0265397 & 0 \\
2 & $\left(g_{0}+4 g_{1}\right) g_{2}$ & 0.0567936 & 0 \\
3 & $\left(g_{0}+4 g_{1}\right) g_{2}$ & 0.00427981 & 0 \\
4 & $\left(g_{0}+4 g_{1}\right) g_{2}$ & -0.0179801 & 0 \\
5 & $\left(g_{0}+4 g_{1}\right) g_{2}$ & 0.0194067 & 0 \\
6 & $\left(3 g_{0}+7 g_{1}\right) g_{2}$ & -0.00663493 & 0 \\
7 & $\left(3 g_{0}+7 g_{1}\right) g_{2}$ & 0.0302668 & -0.046875 \\
8 & $\left(3 g_{0}+7 g_{1}\right) g_{2}$ & 0.00345401 & -0.015625 \\
9 & $\left(3 g_{0}+7 g_{1}\right) g_{2}$ & 0.0309125 & -0.046875 \\
10 & $\left(3 g_{0}+7 g_{1}\right) g_{2}$ & 0.0141984 & 0 \\
11 & $\left(3 g_{0}+7 g_{1}\right) g_{2}$ & -0.00482275 & -0.015625 \\
12 & $\left(3 g_{0}+7 g_{1}\right) g_{2}$ & -0.0810842 & 0.09375 \\
13 & $\left(3 g_{0}+7 g_{1}\right) g_{2}$ & -0.0128297 & 0.03125 \\
14 & $\left(3 g_{0}+7 g_{1}\right) g_{2}$ & -0.00899006 & 0 \\
15 & $\left(3 g_{0}+7 g_{1}\right) g_{2}$ & -0.0132699 & 0 \\
16 & $\left(3 g_{0}+7 g_{1}\right) g_{2}$ & -0.0359603 & 0 \\
17 & $\left(3 g_{0}+7 g_{1}\right) g_{2}$ & 0.00427981 & 0 \\
18 & $\left(3 g_{0}+7 g_{1}\right) g_{2}$ & 0.0359603 & 0 \\
19 & $g_{2}^{2}$ & -0.00706539 & 0 \\
20 & $g_{2}^{2}$ & 0.0201538 & 0 \\
21 & $g_{2}^{2}$ & 0.0161231 & 0 \\
22 & $g_{2}^{2}$ & -0.0208333 & 0 \\
23 & $\frac{\left(g_{0}+9 g_{1}\right) g_{2}^{2}}{g_{1}}$ & 0.00229571 & -0.00195313 \\
24 & $\frac{\left(g_{0}+9 g_{1}\right) g_{2}^{2}}{g_{1}}$ & -0.00400265 & 0.00390625 \\
25 & $\frac{\left(g_{0}+9 g_{1}\right) g_{2}^{2}}{g_{1}}$ & 0.00146635 & -0.00195313 \\
26 & $\frac{\left(g_{0}+9 g_{1}\right) g_{2}^{2}}{g_{1}}$ & -0.00542079 & 0.00390625 \\
27 & $g_{2}^{2}$ & -0.010326 & 0 \\
\hline
\end{tabular}

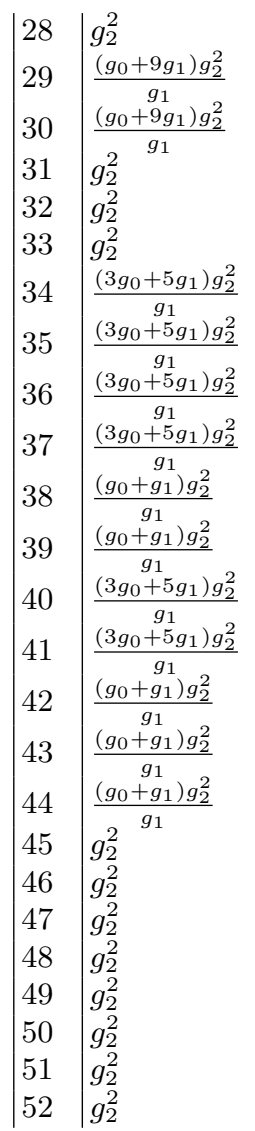

$\left|\begin{array}{l|l}0.00986803 & -0.0117188 \\ -0.000328527 & 0.00390625 \\ 0.00953951 & -0.0078125 \\ 0.0261324 & 0 \\ -0.0207763 & -0.0117188 \\ -0.0234102 & 0.0234375 \\ 0.00229571 & -0.00195313 \\ 0.00146635 & -0.00195313 \\ -0.00542079 & 0.00390625 \\ -0.00400265 & 0.00390625 \\ -0.00344199 & 0 \\ 0.00328934 & -0.00390625 \\ -0.000328527 & 0.00390625 \\ 0.00953951 & -0.0078125 \\ -0.00692543 & -0.00390625 \\ 0.00871079 & 0 \\ -0.00780339 & 0.0078125 \\ 0.00459143 & -0.00390625 \\ -0.00344199 & 0 \\ -0.00800529 & 0.0078125 \\ 0.00328934 & -0.00390625 \\ 0.00293269 & -0.00390625 \\ -0.0108416 & 0.0078125 \\ -0.000657054 & 0.0078125 \\ -0.00692543 & -0.00390625\end{array}\right|$




\begin{tabular}{|l|l|l|l|}
53 & $g_{2}^{2}$ & 0.00871079 & 0 \\
54 & $g_{2}^{2}$ & 0.019079 & -0.015625 \\
55 & $g_{2}^{2}$ & -0.00780339 & 0.0078125 \\
56 & $\frac{\left(g_{0}+3 g_{1}\right) g_{2}^{2}}{g_{1}}$ & -0160893 & 0 \\
57 & $\frac{\left(g_{0}+3 g_{1}\right) g_{2}^{2}}{g_{1}}$ & 0.0123075 & 0 \\
58 & $\frac{\left(g_{0}+3 g_{1}\right) g_{2}^{2}}{g_{1}}$ & 0.00293269 & -0.00390625 \\
59 & $\frac{\left(g_{0}+3 g_{1}\right) g_{2}^{2}}{g_{1}}$ & 0.00459143 & -0.00390625 \\
60 & $\frac{\left(g_{0}+3 g_{1}\right) g_{2}^{2}}{g_{1}}$ & -0.0108416 & 0.0078125 \\
61 & $\frac{\left(g_{0}+5 g_{1}\right) g_{2}^{2}}{g_{1}}$ & 0.00459143 & -0.00390625 \\
62 & $\frac{\left(g_{0}+5 g_{1}\right) g_{2}^{2}}{g_{1}}$ & -0.00800529 & 0.0078125 \\
63 & $\frac{\left(g_{0}+5 g_{1}\right) g_{2}^{2}}{g_{1}}$ & -0.000192854 & 0.0078125 \\
64 & $\frac{\left(g_{0}+5 g_{1}\right) g_{2}^{2}}{g_{1}}$ & 0.00208369 & -0.0078125 \\
65 & $\frac{\left(g_{0}+5 g_{1}\right) g_{2}^{2}}{g_{1}}$ & -0.00344199 & 0 \\
66 & $\frac{\left(g_{0}+5 g_{1}\right) g_{2}^{2}}{g_{1}}$ & 0.00328934 & -0.00390625 \\
67 & $g_{0}^{2}+6 g_{1} g_{0}+10 g_{1}^{2}$ & -0.0359603 & 0 \\
68 & $g_{0}^{2}+6 g_{1} g_{0}+10 g_{1}^{2}$ & 0.127185 & -0.25 \\
69 & $g_{0}^{2}+6 g_{1} g_{0}+10 g_{1}^{2}$ & -0.00832817 & -0.125 \\
70 & $g_{0}^{2}+6 g_{1} g_{0}+10 g_{1}^{2}$ & 0.0635923 & -0.125 \\
71 & $g_{0}^{2}+6 g_{1} g_{0}+10 g_{1}^{2}$ & -0.107881 & 0 \\
72 & $2 g_{0}^{2}+9 g_{1} g_{0}+10 g_{1}^{2}$ & 0.0625 & 0 \\
73 & $2 g_{0}^{2}+9 g_{1} g_{0}+10 g_{1}^{2}$ & 0.0625 & 0 \\
74 & $\frac{\left(g_{0}^{2}+4 g_{1} g_{0}+6 g_{1}^{2}\right) g_{2}}{g_{1}}$ & 0.0157615 & -0.015625 \\
75 & $\frac{\left(g_{0}^{2}+8 g_{1} g_{0}+18 g_{1}^{2}\right) g_{2}}{g_{1}}$ & -0.00378173 & 0 \\
76 & $\frac{\left(g_{0}^{2}+4 g_{1} g_{0}+6 g_{1}^{2}\right) g_{2}}{g_{1}}$ & -0.022533 & 0.03125 \\
77 & $\frac{\left(g_{0}^{2}+4 g_{1} 1 g_{0}+6 g_{1}^{2}\right) g_{2}}{g_{1}}$ & 0.00156315 & -0.015625 \\
78 & $\frac{\left(g_{0}^{2}+8 g_{1}^{2}\right) g_{2}}{\left.g_{1}^{2}\right) g_{2}}$ & -0.00378173 & 0 \\
79 & $\frac{\left(g_{0}^{2}+8 g_{1}\right) g_{2}}{g_{1}}$ & 0.00156315 & -0.015625 \\
80 & $\frac{\left(g_{0}^{2}+8 g_{1}^{2}\right) g_{2}}{g_{1}}$ & -0.022533 & 0.03125 \\
81 & $\frac{\left(g_{0}^{2}+8 g_{1}^{2}\right) g_{2}}{g_{1}}$ & 0.0157615 & -0.015625 \\
82 & $\left(g_{0}+3 g_{1}\right) g_{2}$ & 0.131256 & -0.140625 \\
83 & $\left(g_{0}+3 g_{1}\right) g_{2}$ & -0.00208527 & 0.046875
\end{tabular}

\begin{tabular}{|c|c|c|c|}
\hline 84 & $\frac{\left(g_{0}^{2}+5 g_{1} g_{0}+6 g_{1}^{2}\right) g_{2}}{g_{1}}$ & 0.015625 & 0 \\
\hline & $\frac{\left(g_{0}^{2}+5 g_{1} g_{0}+6 g_{1}^{2}\right) g_{2}}{g_{1}}$ & -0.015625 & 0 \\
\hline & 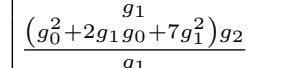 & 0.051701 & -0.0625 \\
\hline & 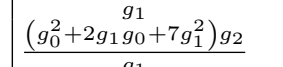 & -0.051701 & 0.0625 \\
\hline & $\left(g_{0}+3{ }^{g_{1}} g_{1}\right) g_{2}$ & 0.126092 & $\begin{array}{l}0.0020 \\
-0.125\end{array}$ \\
\hline & $\left(g_{0}+3 g_{1}\right) g_{2}$ & -0.0719206 & 0 \\
\hline & $\left(g_{0}+3 g_{1}\right) g_{2}$ & -0.0609641 & 0.09375 \\
\hline & $\left(g_{0}+3 g_{1}\right) g_{2}$ & -0.0901321 & 0.125 \\
\hline & $\left(g_{0}+3 g_{1}\right) g_{2}$ & 0.0719206 & 0 \\
\hline & $\left(2 g_{0}+5 g_{1}\right) g_{2}$ & 0.0304821 & -0.046875 \\
\hline & $\left(2 g_{0}+5 g_{1}\right) g_{2}$ & 0.0437519 & -0.046875 \\
\hline & $\left(2 g_{0}+5 g_{1}\right) g_{2}$ & 0.0359602 & 0 \\
\hline & $\frac{\left(g_{0}^{2}+2 g_{1} g_{0}+7 g_{1}^{2}\right) g_{2}}{g_{1}}$ & 0.0100889 & -0.015625 \\
\hline & $\frac{\left(g_{0}^{2}+2 g_{1} g_{0}+7 g_{1}^{2}\right) g_{2}}{g_{1}}$ & 0.00345401 & -0.015625 \\
\hline & $\frac{\left(g_{0}^{2}+2 g_{1} g_{0}+7 g_{1}^{2}\right) g_{2}}{g_{1}}$ & -0.00663493 & 0 \\
\hline & $\frac{\left(g_{0}^{2}+2 g_{1} g_{0}+7 g_{1}^{2}\right) g_{2}}{g_{1}}$ & -0.00690807 & 0.03125 \\
\hline 00 & $\frac{\left(g_{0}+5 g_{1}\right) g_{2}^{2}}{g_{1}}$ & 0.0148442 & -0.0117188 \\
\hline & & -0.0757955 & 0.0703125 \\
\hline & $g_{2}^{2}$ & 0.0549622 & -0.0703125 \\
\hline 103 & $g_{2}^{2}$ & -0.00702507 & $\mid 0$ \\
\hline & & -0.0030681 & 0.0234375 \\
\hline 105 & $\frac{\left(g_{0}+3 g_{1}\right) g_{2}^{2}}{g_{1}}$ & 0.0148442 & -0.0117188 \\
\hline 106 & $\frac{\left(g_{0}+3 g_{1}\right) g_{2}^{2}}{g_{1}}$ & 0.0148442 & -0.0117188 \\
\hline 107 & $\frac{\left(g_{0}+3 g_{1}\right) g_{2}^{2}}{g_{1}}$ & -0.0378978 & 0.0351563 \\
\hline 108 & $\frac{\left(g_{0}+3 g_{1}\right) g_{2}^{2}}{g_{1}}$ & -0.0179801 & 0 \\
\hline 109 & $\frac{\left(g_{0}+3 g_{1}\right) g_{2}^{2}}{g_{1}}$ & -0.0378978 & 0.0351563 \\
\hline & $g_{2}^{2}$ & 0.0113452 & 0 \\
\hline & $g_{2}^{2}$ & -0.0113452 & 0 \\
\hline & $g_{2}^{2}$ & -0.0141308 & 0 \\
\hline 3 & $g_{2}^{2}$ & 0.0120923 & 0 \\
\hline 114 & $\frac{\left(g_{0}+3 g_{1}\right) g_{2}^{2}}{g_{1}}$ & 0.0274811 & -0.0351563 \\
\hline 5 & $\frac{\left(g_{0}+3 g_{1}\right) g_{2}^{2}}{g_{1}}$ & 0.0217619 & 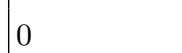 \\
\hline & $3 g_{0}^{2}+3 g_{1} g_{0}+11 g_{1}^{2}$ & 0.144304 & -0.25 \\
\hline
\end{tabular}

Collecting the divergences from all the above diagrams and applying the renormalization condition (13) yields,

$$
\begin{aligned}
Z_{1} & =1+\frac{1}{\epsilon}\left(\lambda_{0}+2.5 \lambda_{1}-0.25 \lambda_{0}^{2}-2.5 \lambda_{1}^{2}-1.5 \lambda_{0} \lambda_{1}+0.0676406 \lambda_{2} \lambda_{0}+0.253861 \lambda_{1} \lambda_{2}+0.00136604 \lambda_{2}^{2}\right. \\
& \left.-\frac{0.0179802 \lambda_{2} \lambda_{0}^{2}}{\lambda_{1}}-\frac{0.000747121 \lambda_{2}^{2} \lambda_{0}}{\lambda_{1}}\right)+\frac{1}{\epsilon^{2}}\left(1.25 \lambda_{0}^{2}+7.75 \lambda_{1}^{2}+3.75 \lambda_{1} \lambda_{0}+0.1875 \lambda_{2} \lambda_{0}+0.46875 \lambda_{1} \lambda_{2}\right)
\end{aligned}
$$

All the results obtained in Appendices B to $\mathrm{I}$ using the computational method described in Appendix $\mathrm{A}$ when truncated to one-loop order agree with the results obtained in Ref. [21, where calculations were performed only to this order.

[1] G. Ódor, Rev. Mod. Phys. 76, 663 (2004)

[2] U. C. Täuber, Critical dynamics: a field theory ap- proach to equilibrium and non-equilibrium scaling behavior (Cambridge University Press, 2014). 
[3] N. Antonov, N. Gulitskiy, P. Kakin, and V. Serov, arXiv preprint arXiv:2005.04756 (2020).

[4] J. T. Young, A. V. Gorshkov, M. Foss-Feig, and M. F. Maghrebi, Physical Review X 10, 011039 (2020).

[5] B. Schmittmann and R. K. Zia, Phase transitions and critical phenomena 17, 3 (1995).

[6] U. C. Täuber, J. E. Santos, and Z. Rácz, The European Physical Journal B-Condensed Matter and Complex Systems 7, 309 (1999).

[7] W. Kinzel, R Zallen and J Adler (Bristol: Hilger) (1983).

[8] H.-K. Janssen, Zeitschrift für Physik B Condensed Matter 42, 151 (1981).

[9] S.-K. Ma, Modern theory of critical phenomena (Routledge, 2018).

[10] P. C. Hohenberg and B. I. Halperin, Reviews of Modern Physics 49, 435 (1977).

[11] S. Katz, J. L. Lebowitz, and H. Spohn, Physical Review B 28, 1655 (1983).

[12] S. Katz, J. L. Lebowitz, and H. Spohn, Journal of statistical physics 34, 497 (1984).

[13] D. Helbing, Reviews of modern physics 73, 1067 (2001).

[14] D. Chowdhury, L. Santen, and A. Schadschneider, Phys. Repts 329, 4.

[15] L. Sieberer, S. D. Huber, E. Altman, and S. Diehl, Physical Review B 89, 134310 (2014).

[16] U. C. Täuber and S. Diehl, Physical Review X 4, 021010 (2014).

[17] H. Vuijk, R. Rens, M. Vahabi, F. MacKintosh, and A. Sharma, Physical Review E 91, 032143 (2015).
[18] H. Janssen and B. Schmittmann, Zeitschrift für Physik B Condensed Matter 64, 503 (1986).

[19] B. Schmittmann and K. Bassler, Physical review letters 77, 3581 (1996).

[20] K. Bassler and B. Schmittmann, Physical Review E 49, 3614 (1994).

[21] S. B. Dutta and S.-C. Park, Phys. Rev. E 83, 011117 (2011)

[22] K.-t. Leung and J. L. Cardy, Journal of statistical physics 44, 567 (1986).

[23] V. Becker and H. Janssen, EPL (Europhysics Letters) 19, 13 (1992).

[24] P. C. Martin, E. Siggia, and H. Rose, Physical Review A 8, 423 (1973).

[25] T. Hahn, Computer Physics Communications 140, 418 (2001).

[26] R. Mertig, M. Böhm, and A. Denner, Computer Physics Communications 64, 345 (1991).

[27] V. Shtabovenko, R. Mertig, and F. Orellana, Computer Physics Communications 207, 432 (2016).

[28] W. R. Inc., "Mathematica, Version 11.1," Champaign, IL (2017).

[29] J. Carter and G. Heinrich, Computer Physics Communications 182, 1566 (2011).

[30] W. Bruns, B. Ichim, T. Römer, R. Sieg, and C. Söger, "Normaliz. algorithms for rational cones and affine monoids," (2010).

[31] W. Bruns, B. Ichim, and C. Söger, arXiv preprint arXiv:1206.1916 (2012). 